\title{
Emergence of Innovative Properties by Replacement of Nitrogen Atom with Phosphorus Atom in Quaternary Ammonium Ionic Liquids: Insights from ab initio Calculations and MD Simulations
}

\author{
Mohammad Hadi Ghatee*, Maryam Bahrami \\ (Department of Chemistry, Shiraz University, Shiraz 71946, Iran)
}

E-mail: ghatee@ susc.ac.ir, mhghatee2@gmail.com

Fax: +98 7136460788 , Tel: +98 7136137174

* corresponding Author 


\section{Table of Content}

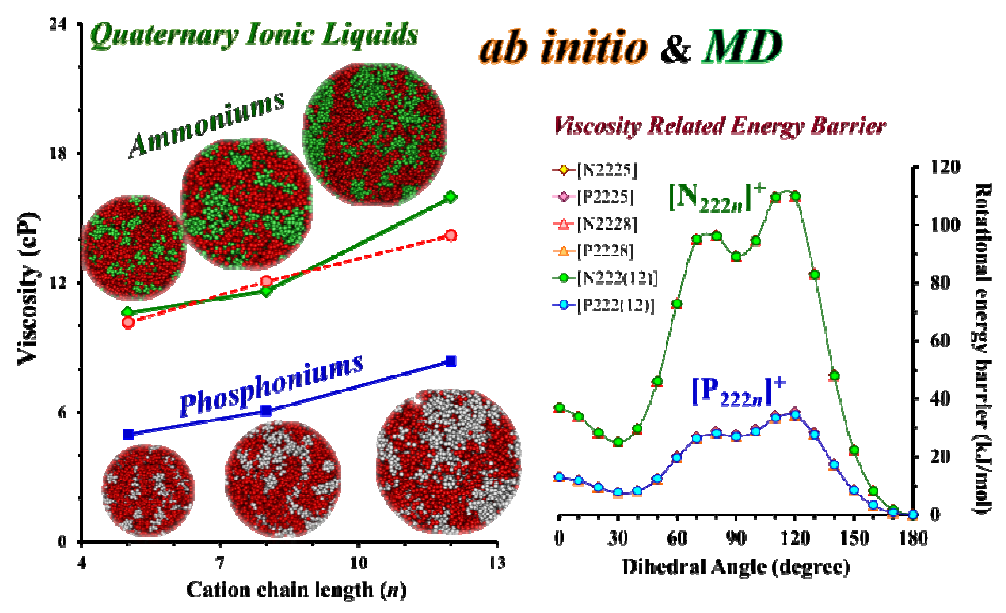




\begin{abstract}
We investigate to contrasting structure, dynamic and thermophysical properties of quaternary ammonium and phosphonium ionic liquids (ILs) based on triethylalkylammonium $\left[\mathrm{N}_{222 n}\right]^{+}$and triethylalkylphosphonium $\left[\mathrm{P}_{222 n}\right]^{+}$cations $(n=5,8,12)$ and (bis(trifluoromethylsulfonyl)imide) anion $\left[\mathrm{NTf}_{2}\right]^{-}$by quantum chemical calculations (QCC) and molecular dynamics (MD) simulations. QCCs conform to previous studies, showing that phosphonium cation alkyl chain rotational-energy-barrier is lower than ammonium cation. These molecular nature leads to no appreciable differences in their liquid density. However, their simulated transport properties (self-diffusion, conductivity, etc) are appreciably different. In particularly, viscosity of phosphoniums are much lower than ammoniums. Ammoniums make nano-scale structural domains larger than phosphoniums. Employed analysis, vector re-orientational dynamics, ion-pair lifetime and nanostructure domain are in favor of faster dynamic for phosphoniums than ammoniums. [ $\left.\mathrm{NTf}_{2}\right]^{-}$anion features a long lived pairing with ammoniums than phosphoniums. Overall, phosphoniums possess higher transference number, higher conductivity, and appreciably lower viscosity favorable for higher electrochemical performances.
\end{abstract}

Keywords: Ammonium and phosphonium ionic liquids; Molecular dynamics and computational chemistry; Interionic dynamics analysis; Nanostructural domains; Thermodynamic and transport properties. 


\section{INTRODUCTION}

Room-temperature ionic liquids (RTILs) are organic salts which are liquid at or near ambient temperature. They are composed of low-symmetrical bulky organic cations among which imidazolium's are the most well-known and well-characterized. Contrary to inorganic ionic compounds with high lattice energy, cations are poorly associated to variety of organic or inorganic anions like halides, $\left[\mathrm{PF}_{6}\right]^{-},\left[\mathrm{BF}_{4}\right]^{-},\left[\mathrm{N}(\mathrm{CN})_{2}\right]^{-},\left[\mathrm{NTf}_{2}\right]^{-}$, etc. Unique properties shown by ILs are originated from ionic nature, relative structure, and particular anion-cation interaction. Selection of anion with variety of cation could lead to IL with physicochemical properties desired in chemistry, physics, biology, industry. ${ }^{[1-11]}$ Thermodynamic properties smoothly but transport properties sharply changes with the combination of cation-anion, which challenges research works and applications of ILs.

Aprotic room temperature ILs based on quaternary cations, such as quaternary ammoniums (QA) and quaternary phosphoniums (QP) exhibit interesting properties compared to ionic liquids based on heterocyclic cations. For example, QA and QP cations combined with $\left[\mathrm{NTf}_{2}\right]^{-}$anion to form hydrophobic ILs have wide electrochemical windows and better thermal and chemical stability in comparison with pyridinium and imidazolium-based ILs. ${ }^{[12]}$ QA and QP based ILs have been known and produced for years, but neglected in literature compared to other types of ILs. Significant applications as solvent, lubricant and nanowire-based lithium ion batteries have been cited for these ILs. ${ }^{[13-17]}$ Aprotic QAILs and QPILs with $\left[\mathrm{NTf}_{2}\right]^{-}$anion are different in density, viscosity, melting temperature, enthalpy, ionic conductivity, electrochemical stability and hydrophobicity. QPILs exhibit higher mobility (due to lower viscosity), thermal stability and conductivity than QAILs analogues. Accordingly, QPILs are good candidates of use in electrochemical applications, such as voltametric measurements for various redox couples, ${ }^{[18]}$ anodic polymerization of pyrrole, ${ }^{[19]}$ supercapacitors, ${ }^{[20]}$ dye-sensitized solar cells ${ }^{[21,22]}$ and high temperature reactions. ${ }^{[23]}$ QPILs are valuable low-viscosity solvents and proved suitable for many specific applications. ${ }^{[24-39]}$ Industrial design and lab application requires enough information about their thermophysical properties. Properties such as melting temperature, density, viscosity, conductivity, surface tension, gas solubility, refractive index, and critical temperature found to be optimally useful. Macroscopic properties of ILs, like viscosity or ionic conductivity, have been largely investigated. However, a profound understanding of the relation between these macroscopic properties and the microscopic structure of cations and anions is still demanding. Various experimental and theoretical techniques have been used to study the thermophysical properties of ILs based on QA and QP cations with $\mathrm{NTf}_{2}{ }^{-}$anion. ${ }^{[23,40-54]}$ Several reports confirmed lower viscosities and higher conductivities of QPILs than QAILs counterparts. ${ }^{[23,44,48,55]}$ The 
question arises here is how and to what extent the properties of QAILs are different from those of QPILs. Obviously, the reason for the difference in their properties can be understood by investigation of the electronic and geometrical structure of cation including the central atom, which is achievable by the QCC methods.

Molecular dynamics (MD) simulations method based on classical force fields is a powerful technique to study the thermodynamics, structure and dynamics of ionic liquids at an atomistic and molecular level, with the outcome critically comparable with experiment. However, the success of simulation is guaranteed only by highly accurate force field, particularly the simulation of transport properties, making MD technique challenging. It is known that a polarizable force field reproduces the dynamic properties quite accurately. Polarization is either modeled by a polarizable force field or represented by a nonpolarizable force field modified by implicit treatment of polarization via reducing net charge, which requires much experimental information to parameterize the force field. Lopes and Pádua, ${ }^{[56]}$ proposed a set of force field parameters for the simulation of ionic liquids containing $\mathrm{CF}_{3} \mathrm{SO}_{3}{ }^{-}$( $\left.\mathrm{Tf}^{-}\right)$and $\left[\mathrm{NTf}_{2}\right]^{-}$anions, producing accurate crystal structures and liquid density.

Some research groups have investigated thermodynamics, structure, and dynamic properties of QAILs and QPILs including [NTf $]^{-}$anion by MD simulations. ${ }^{[57-68]}$ Only a few systematic studies have directed to investigate how switching from $\mathrm{N}$ atom to $\mathrm{P}$ atom influences the physicochemical properties. ${ }^{[44,47,48,55]}$ However, little is known about ionic interactions in these ILs. Under such circumstances, there is a twofold question: first, what are the differences between liquid microstructure of QAILs and QPILs, and second, how differences in liquid microstructure of these ILs affect their physicochemical properties?

Recently, Scarbath-Evers et al. ${ }^{[55]}$ studied molecular features contributing to the lower viscosity of phosphonium-based ILs with chloride anion $\left(\left[\mathrm{P}_{2228}\right] \mathrm{Cl}\right)$ compared to ammoniums $\left(\left[\mathrm{N}_{2228}\right] \mathrm{Cl}\right)$ by static quantum chemistry calculations and classical molecular dynamics simulations. They have considered atomic size, bond length, and energy barrier to the rotational states in relation to the cations. Their simulated viscosity (by Green-Kubo method), as the representation of the transport property, has involved high deviation from experimental data and not published. The viscosity of ILs involving chloride anion are generally extremely high, unfavorable for accurate simulation of dynamic properties, and may account for such high deviation. On the contrary, ILs involving $\left[\mathrm{NTf}_{2}\right]^{-}$anion lead to moderate viscosity. In spite of the above overview representing works done for the properties of both type of ILs by experiments and simulations, to date no abundant MD simulation investigation has been performed to study comparatively the physicochemical and structural properties of QAILs with QPILs involving $\left[\mathrm{NTf}_{2}\right]^{-}$anion as a function of temperature. 
We perform a comprehensive study on structural, thermodynamical, and dynamical properties of isostructural RTILs based on triethylalkylammonium $\left[\mathrm{N}_{222 n}\right]^{+}$and triethylalkylphosphonium $\left[\mathrm{P}_{222 n}\right]^{+}$ cations (with $n=5,8,12$ ) and [ $\left.\mathrm{NTf}_{2}\right]^{-}$(bis(trifluoromethylsulfonyl)imide) anion at atomistic level using QCCs and classical MD simulations. The nonpolaraizable all-atom OPLS force field is applied ${ }^{[56,59]}$ The impact of cation core atom on the difference in properties particularly the lower viscosity of QPILs over the QAILs is center of attention.

In Section 2 computational details, electronic structure calculation by QCC, force field and MD simulation details are presented. The results of QCC for partial atomic charges and rotational energy barriers are given in section 3. In the following subsections the simulated thermodynamic and transport properties will be discussed. Further analysis like vector re-orientational dynamics, ion-pair lifetime, and nano-structure domains are employed to detail more the anion-cation features. Long live ion-pairing feature of $\left[\mathrm{NTf}_{2}\right]^{-}$anion with different cations will be discussed. The section 4 is devoted to conclusions and remarks.

\section{COMPUTATIONAL DETAILS}

Computations comprise two parts. First the density functional theory (DFT) is used to calculate properties related to geometrical and electronic structure of the ILs molecules in gas phase, and next thermodynamic and dynamic properties are simulated by using classical molecular dynamics.

\subsection{Electronic Structure (DFT Calculations)}

First anions and cations were optimized and then brought together to the most probable orientation. The structure of each IL ion-pair was primarily optimized by classical molecular mechanics simulation at $10 \mathrm{~K}$, and finally was optimized by DFT at B3LYP/6-31+G(2df,p $)^{[69]}$ level of theory (applied to all atoms), using GAUSSIAN 03 program. ${ }^{[70]}$ Optimizing the ion-pair, particularly for these ILs that are known to be in the form of ion-pair, produces more realistic electrostatic terms. (Figure 1) (See supporting information for energies (Table SI1), structures of different conformers of cations; energies (Table SI2) of all optimized ILs ion-pairs with regards to the cis and trans anions; see also Figures in Tables SI3, SI4, and SI5 for atom labelling) Absence of imaginary harmonic vibrational frequencies confirms the stable structures were produced. ${ }^{[71]}$

The atomic charges for all ILs ion-pairs were taken from DFT calculations obtained by using electrostatic potential fitting (CHELPG) procedure implemented in GAUSSIAN 03. Therefore, more realistic electrostatic terms are used in all simulation force fields. ${ }^{[72]}$ In agreement with literature ${ }^{[73,74]}$ the sum of calculated atomic charges of cation and anion for each IL is less than unity with net-zero (see Table 1 Part A). 


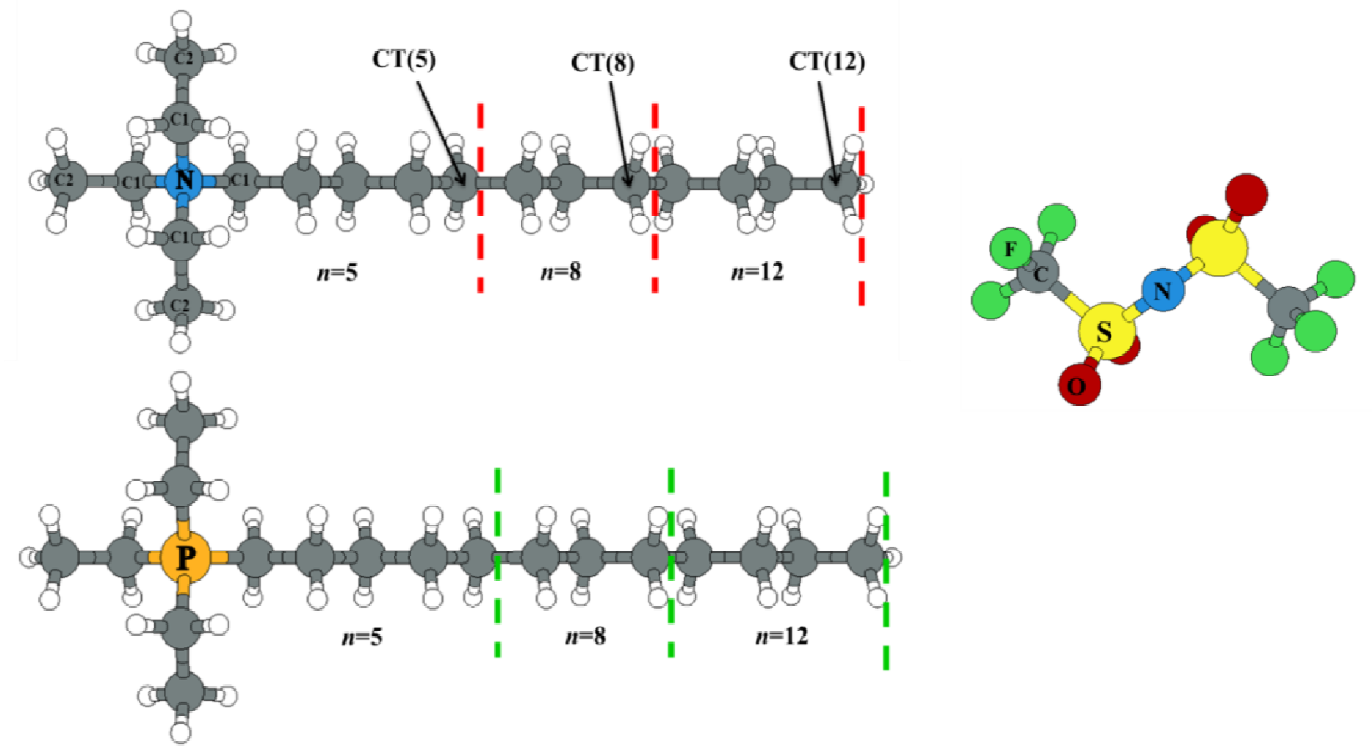

Figure 1. Structure of quaternary ammonium and phosphonium cations and $\left[\mathrm{NTf}_{2}\right]^{-}$anion used for molecular dynamics simulations.

Considering the hopping mechanism of the particle mobility, the probability of having different conformer of quaternary cations strongly influences transport properties of the ILs. Hence, we calculated the rotational energy barriers of some selected cation and ion-pair conformers at B3LYP/6$31+\mathrm{G}(2 \mathrm{df}, \mathrm{p})$ and MP2/6-311++G(d,p) level of theory. The rotational energy barrier represents an estimate of the influence of bond length, bond angle and the dihedral angle comparatively when the difference in transport properties of the two type of ILs is concerned. The change in potential energy was calculated every $10^{\circ}$ rotation about $\mathbf{N}-\mathbf{C}$ and $\mathbf{P}-\mathbf{C}$ : single bond of quaternary cations. The results are presented where the total energies are normalized with respect to the energy of corresponding conformer at $180^{\circ}$.

\subsection{Force Field}

The OPLS force field, developed by Canongia Lopes et al. (Eq. 1), was used. ${ }^{[56,59,75-77]}$ The pairwise-additive potential energy, $\boldsymbol{u}_{r . h}$, decomposes into covalent bonds, valence angles, torsion (dihedral) angles, and atom-atom pairwise repulsive, dispersive, and electrostatic contributions: 


$$
\begin{aligned}
& u_{\alpha, \beta}=\sum_{i j}^{\text {bonds }} \frac{k_{i j}}{2}\left(r_{i j}-r_{u, \nu}\right)^{2} \\
&+\sum_{i j k}^{\text {angles }} \frac{\theta_{u j}}{2}\left(\theta_{i j k}-\theta_{0, i j k}\right)^{2} \\
&+\sum_{i j k t}^{\text {aihudrato }} \sum_{m=1}^{4} \frac{V_{m, i j k l}}{2}\left[1-(-1)^{m} \cos \left(m \varphi_{i j k l}\right)\right] \\
&+\sum_{i j}^{\text {nonbonded }}\left\{4 \varepsilon_{i j}\left[\left(\frac{\sigma_{i j}}{r_{i j}}\right)^{12}-\left(\frac{\sigma_{i j}}{r_{i j}}\right)^{6}\right]+\frac{1}{4 \pi s_{0}} \frac{q_{i} q_{j}}{r_{i j}}\right\}
\end{aligned}
$$

where, all terms have their usual meanings. The Coulomb interactions are defined in terms of atomic point charges, using the calculated CHELPG charges. The repulsive and dispersive terms are described by the Lennard-Jones (12-6) potential. Lennard-Jones parameters for unlike pair of atoms were obtained by using the Lorentz-Berthelot mixing rule: ${ }^{[78]} \varepsilon_{i \bar{i}}=\sqrt{\boldsymbol{\varepsilon}_{\overline{i j}} \boldsymbol{\varepsilon}_{i \bar{i}}}$ and $\boldsymbol{\sigma}_{\overline{i j}}=\left[\boldsymbol{\sigma}_{\overline{i j}}+\boldsymbol{\sigma}_{i \bar{i}}\right) / \mathbf{2}$.

The vdW parameters are taken from OPLS force field for ionic liquids developed by Lopes and coworkers and modified by Tsuzuki et al. ${ }^{[59,76]}$ The optimized geometries were used to set equilibrium bond lengths and angles. The bond and angle force constants and dihedral parameters were obtained from the literature. ${ }^{[59,76]}$ (see Tables SI3 to SI9 for force field parameters).

\subsection{Simulation Details}

A single ion-pair of each IL optimized via DFT calculation was replicated to obtain a threedimensional simulation box containing 150 ion-pairs. For each liquid system $\left(\left[\mathrm{N}_{2225}\right] \mathrm{NTf}_{2}\right.$, $\left[\mathrm{N}_{2228}\right] \mathrm{NTf}_{2},\left[\mathrm{~N}_{222(12)}\right] \mathrm{NTf}_{2},\left[\mathrm{P}_{2225}\right] \mathrm{NTf}_{2},\left[\mathrm{P}_{2228}\right] \mathrm{NTf}_{2}$ and $\left.\left[\mathrm{P}_{222(12)}\right] \mathrm{NTf}_{2}\right)$, we performed simulation studies of bulk at ambient pressure $\left(1.01325 \times 10^{5} \mathrm{~Pa}\right)$ using the DL_POLY 2.17 program. ${ }^{\text {[79] }}$

The Verlet-Leapfrog integration algorithm under the periodic boundary conditions was used. The Columbic long-range interactions were calculated using Ewald method with a precision of $1 \times 10^{-5}$. The potential cut-off distance of 19-22 A was used. Starting with a short time step, the simulation then followed by time step of $10^{-3} \mathrm{ps}$. With the initial equilibration at $298 \mathrm{~K}$, the system then underwent a stepwise heating to $420 \mathrm{~K}$ and cooling to $373 \mathrm{~K}$ (in steps of $20 \mathrm{~K}$ ). The simulation was continued for 1 ns at each temperature in NPT ensemble using the Nosé-Hoover thermostat/barostat with relaxation times of 0.5 and 2 . The simulation was then continued at six successive temperatures, back to $298 \mathrm{~K}$.

The density of ILs was evaluated from 4 ns MD trajectories in NPT ensemble after a 3 ns MD equilibration run at each temperature. The transport properties of ILs (self-diffusion coefficients, viscosities and ionic conductivities) were evaluated at $373 \mathrm{~K}$ in $N V T$ ensemble. Table SI10 
demonstrates the melting point of all ILs studied. After a 3 ns equilibration, a 14 ns production run was performed to calculate self-diffusion coefficients of ions from the simulated mean square displacements (MSD). Longer simulation time was required, up to $16 \mathrm{~ns}$, to attain equilibrium for the ensemble of slow dynamic ILs with long alkyl-chain length, i.e., [ $\left.\mathrm{N}_{222(12)}\right] \mathrm{NTf}_{2}$. The size of the simulation boxes are shown in Table SI11.

\section{RESULTS AND DISCUSSION}

Nitrogen and phosphorus atoms of cationic core of QAILs and QPILs are different in size, mass, electronegativity, electronic structure and polarizability. These differences in the nature of cationic central atoms are potentially responsible for any differences in physical, chemical, electrochemical, and thermophysical properties observed for theses ILs.

Thermophysical properties like density, viscosity and ionic conductivity of ILs cannot be obtained simply by QCCs, though they are produced by simulation methods with the accuracy comparable with experiment. The key points here are the accuracy of the method and force field parameters to be able to contrast properties of QAIL's and QPIL's precisely. Basically thermodynamic properties are only slightly sensitive to the nature of compound under consideration, whereas dynamic properties show appreciably high sensitivity.

\subsection{Quantum Chemistry Calculations (QCCs)}

Direct consequents of the replacement of cation central atom ( $\mathrm{N}$ atom by $\mathrm{P}$ atom) determined by QCCs are the shift in atomic charges, the molecular sizes, and the bond length of central atom attached to $\mathrm{C}$ atom. The first two microscopic shifts could affect the thermodynamic properties and all shifts could have influences on the dynamic properties, as will be discussed subsequently. Also the shift in atomic charges should have shift the Coulombic interactions, and hence the molecular energetics.

DFT calculations show a considerable delocalization of atomic charges of the ILs when $\mathrm{N}$ atom is replaced by $\mathrm{P}$ atom of the cation. Charges of $\mathrm{P}$ atom in phosphonium cations are substantially more positive ( $\left.\left[\mathrm{P}_{2225}\right] \mathrm{NTf}_{2}: 0.248 e ;\left[\mathrm{P}_{2228}\right] \mathrm{NTf}_{2}: 0.263 e ;\left[\mathrm{P}_{222(12)}\right] \mathrm{NTf}_{2}: 0.273 e\right)$ than $\mathrm{N}$ atom in ammonium

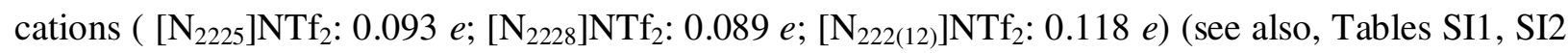
and SI3). These results are in good agreement with the literature. ${ }^{[44,48]}$ Such a redistribution of charge on cation head group would have a profound effect on the anion-cation interaction as well as the degree of nano-aggregation formed by nano-phase segregation of the charge-rich ionic centers from the non-polar alkyl-chains, as will be discussed later. 
Table 1. Comparing, Part A: cation and anion charges (C) of QAILs with QPILs obtained by CHELPG procedure; Part B: N with P atom charges (C) of cation in QAILs and QPILs; Part C: N-C with P-C bond length $(\AA)$ of cation in QAILs and QPILs.

\begin{tabular}{|c|c|c|c|}
\hline \multicolumn{4}{|c|}{ Part A } \\
\hline QAILs & charge & QPILs & charge \\
\hline$\left[\mathrm{N}_{2225}\right] \mathrm{NTf}_{2}$ & \pm 0.968 & {$\left[\mathrm{P}_{2225}\right] \mathrm{NTf}_{2}$} & \pm 0.955 \\
\hline$\left[\mathrm{N}_{2228}\right] \mathrm{NTf}_{2}$ & \pm 0.968 & {$\left[\mathrm{P}_{2228}\right] \mathrm{NTf}_{2}$} & \pm 0.953 \\
\hline$\left[\mathrm{N}_{222(12)}\right] \mathrm{NTf}_{2}$ & \pm 0.964 & {$\left[\mathrm{P}_{222(12)}\right] \mathrm{NTf}_{2}$} & \pm 0.955 \\
\hline \multicolumn{4}{|c|}{ Part B } \\
\hline QAILs & charge on $\mathrm{N}$ atom & QPILs & charge on $\mathrm{P}$ atom \\
\hline$\left[\mathrm{N}_{2225}\right] \mathrm{NTf}_{2}$ & 0.0930 & {$\left[\mathrm{P}_{2225}\right] \mathrm{NTf}_{2}$} & 0.248 \\
\hline$\left[\mathrm{N}_{2228}\right] \mathrm{NTf}_{2}$ & 0.0890 & {$\left[\mathrm{P}_{2228}\right] \mathrm{NTf}_{2}$} & 0.263 \\
\hline$\left[\mathrm{N}_{222(12)}\right] \mathrm{NTf}_{2}$ & 0.118 & {$\left[\mathrm{P}_{222(12)}\right] \mathrm{NTf}_{2}$} & 0.273 \\
\hline \multicolumn{4}{|c|}{ Part C } \\
\hline QAILs & $\mathrm{N}-\mathrm{C}$ bond length & QPILs & P-C bond length \\
\hline$\left[\mathrm{N}_{2225}\right] \mathrm{NTf}_{2}$ & 1.5261 & {$\left[\mathrm{P}_{2225}\right] \mathrm{NTf}_{2}$} & 1.8217 \\
\hline$\left[\mathrm{N}_{2228}\right] \mathrm{NTf}_{2}$ & 1.5261 & {$\left[\mathrm{P}_{2228}\right] \mathrm{NTf}_{2}$} & 1.8215 \\
\hline$\left[\mathrm{N}_{222(12)}\right] \mathrm{NTf}_{2}$ & 1.5264 & {$\left[\mathrm{P}_{222(12)}\right] \mathrm{NTf}_{2}$} & 1.8217 \\
\hline
\end{tabular}

The difference in atomic radius of $\mathrm{N}$ and $\mathrm{P}(\sigma(\mathrm{N}): 3.45 \AA$; $\sigma(\mathrm{P}): 3.74 \AA$ ) leads to different bond length (N-C, $1.526 \AA$; P-C, $1.822 \AA)$ in QAILs and QPILs. These features were obtained by performing QCCs on a single ion-pair of QAIL and QPIL in the gas phase at B3LYP/6-31+G(2df,p) level of theory (Table 1, Part C).

One of the key factors controlling the diffusion of ions in ionic liquids is the conformational flexibility. ${ }^{[\mathbf{8 0 , 8 1 ]}}$ Considering the relative atomic radius of central atom (i.e., $\mathrm{P}>\mathrm{N}$ ) and their relative bond length with carbon atom (i.e., C-P>C-N), a higher flexibility is expected for a given conformation of QPIL than QAIL. The flexibility can be quantified by the bond and the dihedral angles. This can be quantified by estimating the energy required for a conformer to pass through a barrier. As a result, energy barrier to the corresponding rotation in the cation is modified when $\mathrm{N}$ atom is substituted by $\mathrm{P}$ atom of the cation. Therefore, we obtained the potential energy changes during rotation about the N-C and P-C single bond of all quaternary cations and cation-anion ion-pairs. To do so, the relaxed potential energy surface was scanned for dihedral angle $\boldsymbol{\tau}$, e.g., $C^{4}-C^{3}-X-C^{1}(X=N$ and P) in steps of $10^{\circ}$ at B3LYP/6-31+G(2df,p) and MP2/6-311++G(d,p) level of theory. As shown in Figure 2(a), the maximum barriers to the rotation for $\left[\mathrm{N}_{2225}\right]^{+}$and $\left[\mathrm{P}_{2225}\right]^{+}$cation are found to be 110.2 (107.4) and 35.4 (35.2) $\mathrm{kJmol}^{-1}$ at $120^{\circ}$, respectively, where DFT values are shown in parenthesis. 
Thus, replacement of $\mathrm{N}$ by $\mathrm{P}$ in quaternary cation substantially reduces the rotational energy barrier by $74.8(72.2) \mathrm{kJmol}^{-1}$ in the above case. Importantly, it is almost irrespective of the alkyl chain length $75.5(72.8,) \mathrm{kJmol}^{-1}$ for $\left[\mathrm{N}_{2228}\right]^{+}$and $\left[\mathrm{P}_{2228}\right]^{+} ; 75.5(72.9) \mathrm{kJmol}^{-1}$ for $\left[\mathrm{N}_{222(12)}\right]^{+}$and $\left.\left[\mathrm{P}_{222(12)}\right]^{+}\right)$. Therefore, although many conformations are probable, the difference in energy barrier is solely due to replacement of $\mathrm{N}$ by $\mathrm{P}$. The vital conclusion is that more conformational states are accessible to the QPILs than QAILs under the same condition. Therefore, if both QPILs and QAILs are brought under external stress by the same amount, the resulted strain in the former liquid is higher than in the latter. A conformational analysis based on the dihedral angles involving core atom of each ILs series will be given in section 3.4.4.

Other conformers involving ethyl groups, pure long alkyl chain and cation in pair with anion were also considered. Figure 2(c) shows the dihedral potential energies involving the ethyl group. A comparison of Figure 2(a) and 2(c) with 2(d) (in which $\mathrm{N}$ or P atom is absence) strictly substantiates the role of central $\mathrm{P}$ atom on lowering the corresponding rotational energy barriers of QPILs. The energy barrier for all ILs is almost the same: $\sim 12.8 \mathrm{~kJ} / \mathrm{mol}$ at $120^{\circ}$ and $\sim 29.8 \mathrm{~kJ} / \mathrm{mol}$ at $0^{\circ}$ (Figure 2(d)). This notable role of central $\mathrm{P}$ atom is furthermore substantiated when the rotational energy barrier of the cation was considered in the presence of $\left[\mathrm{NTf}_{2}\right]$ anion as shown in Figure 2(b). The difference in the energy barrier of the two type of ILs highly enhances at the dihedral angle $60^{\circ}-70^{\circ}$, and diminishes at the extremes (specially for the anti-conformers), while $\left[\mathrm{N}_{2228}\right] \mathrm{NTf}_{2}$ show some deviations.
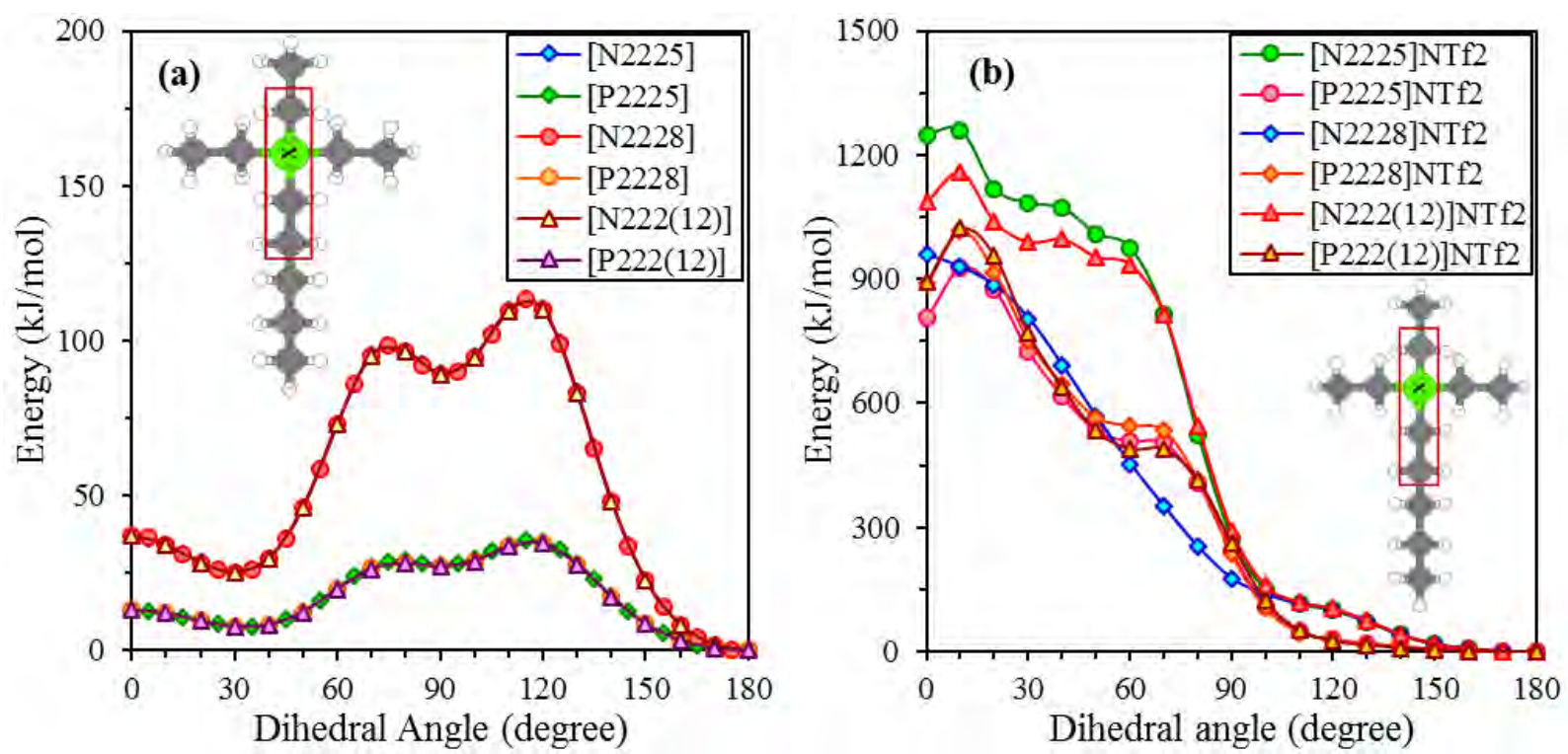

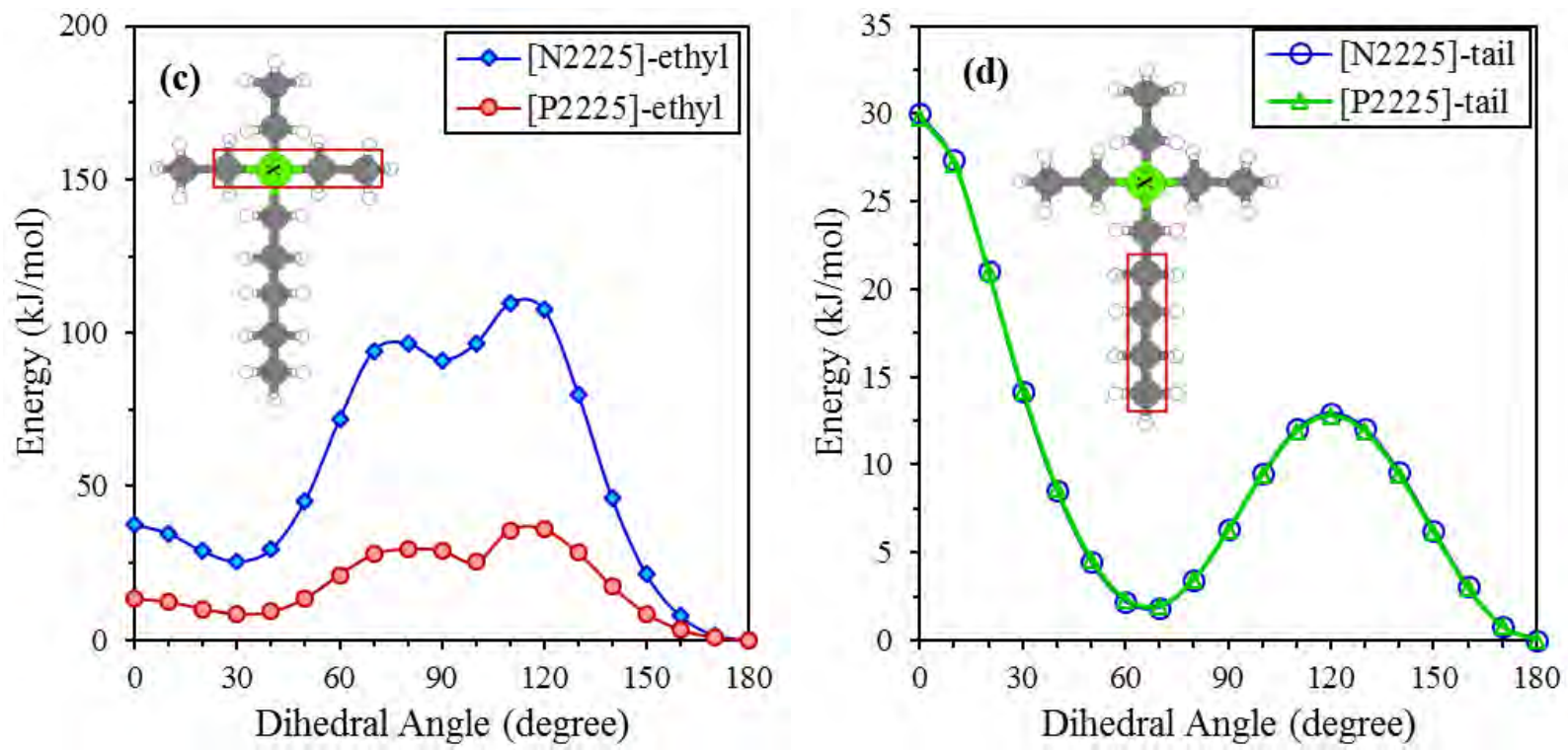

Figure 2. Rotational energy barrier for (a) $\left[\mathrm{X}_{222 n}\right]^{+}$cations and (b) $\left[\mathrm{X}_{222 n}\right] \mathrm{NTf}_{2}$ ion-pairs $(\mathrm{X}=\mathrm{N}, \mathrm{P} ; n=5,8,12)$ calculated at MP2/6-311++G(d,p) level of theory. No appreciable differences are seen compare to the calculation based on B3LYP/6-311++G(d,p) (see text for numerical comparison). (c) when ethyl head group C atoms and $\mathrm{N}$ or $\mathrm{P}$ atom of $\left[\mathrm{X}_{222 n}\right]^{+}$cations are involved. (d) when $\mathrm{N}$ or $\mathrm{P}$ atoms of the $\left[\mathrm{X}_{222 n}\right]^{+}$cations are not involved. Inserts show the specific atoms involved in each calculations. Lines are trend lines

Cation-anion interaction energies $\left(E_{\text {int. }}\right)$, deformation energies $\left(E_{\text {def. }}\right)$ and stabilization energies ( $E_{\text {form. }}$ ) of QAILs and QPILs were calculated at B3LYP/6-31+G(2df,p) level of theory by utilizing Eqs. (2) and (3):

$$
\begin{aligned}
& E_{i n t}=E_{i n n m i r}-\left(E_{r n t i n m}+E_{m m i n m}\right)
\end{aligned}
$$

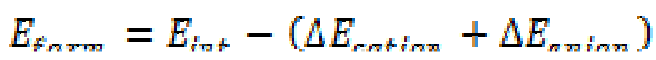

As shown in Table 2, $E_{\text {int. }}$ of both series of ILs increase slightly with cation chain length (see Table 2). In both series of ILs, $E_{\text {def. }}$ and $E_{\text {form. }}$ are increased with cation chain length; at all cation chain lengths, QAILs have smaller $E_{\text {def. }}$ and $E_{\text {form. }}$ than QPILs.

Based on calculated gas-phase formation energies, cation-anion interaction in QAILs is only slightly stronger than QPILs. $E_{\text {int. }}$ values for the two types of ILs are very close to one another and therefore they can't be used as a criteria for strict contrasting of the desired properties, and particularly do not represent the marked lower viscosity of the phosphoniums over the ammoniums.

Table 2. Values of cation-anion interaction energies $\left(E_{\text {int }}\right)$, stabilization energies $\left(E_{\text {form }}\right)$ and deformation energies $\left(E_{\mathrm{def}}\right)$ calculated for QAILs and QPILs at B3LYP/6-31+G(2df,p) level of theory. All calculated energies are in $\mathrm{kJmol}^{-1}$. 


\begin{tabular}{lccc}
\hline \multicolumn{1}{c}{$\mathrm{ILs}$} & $E_{\text {int. }}$ & $E_{\text {form }}$ & $E_{\text {def. }}$ \\
\hline$\left[\mathrm{N}_{2225}\right] \mathrm{Tf}_{2} \mathrm{~N}$ & -278.96 & -273.88 & 5.08 \\
{$\left[\mathrm{~N}_{2228}\right] \mathrm{Tf}_{2} \mathrm{~N}$} & -278.58 & -273.45 & 5.12 \\
{$\left[\mathrm{~N}_{222(12)}\right] \mathrm{Tf}_{2} \mathrm{~N}$} & -278.06 & -272.92 & 5.14 \\
{$\left[\mathrm{P}_{2225}\right] \mathrm{Tf}_{2} \mathrm{~N}$} & -279.23 & -273.48 & 5.75 \\
{$\left[\mathrm{P}_{2228}\right] \mathrm{Tf}_{2} \mathrm{~N}$} & -278.29 & -272.51 & 5.78 \\
{$\left[\mathrm{P}_{222(12)}\right] \mathrm{Tf}_{2} \mathrm{~N}$} & -278.16 & -271.91 & 6.25 \\
\hline
\end{tabular}

\section{Molecular Dynamics Simulation}

3.2.1 Thermodynamic Properties. Liquid density is the most relevant quantity in thermodynamics of liquids. It is related to mass, size and interparticle interactions of constituting atom. Density plays an essential role in extractions, design of energetic compounds, and flotation of fluids used in inertial instruments.

For ILs under study, we simulated densities in the temperature range 298 to $373 \mathrm{~K}$ and 1 atm pressure, using original partial atomic charges determined by our DFT calculations. As plotted in Figure 3 and compared with experimental values in Table SI12, the simulated density decreases quite linearly with temperature for both series of ILs. As it can be seen in Table SI12, the simulated values of $\left[\mathrm{N}_{222 n}\right] \mathrm{NTf}_{2}$ densities and their trend with temperature are in good agreement with our pervious experimental data, ${ }^{[46]}$ (max. dev.=0.78\%). To our knowledge a limited number of experimental densities data of these QPILs have been reported in literature.

In both series of ILs, the simulated density decreases with increasing alkyl-chain length of cation, in good agreement with experimental results but slightly lower than experimental values at all temperatures. As a result, QAILs have slightly higher densities than QPILs counterparts in agreement with experimental density at $298 \mathrm{~K}$ for QPIL. ${ }^{[23,43,44]}$ Exception is for the simulation of long alkyl-chain, where the order of density is reversed, i.e., $\left[\mathrm{P}_{222(12)}\right] \mathrm{NTf}_{2}>\left[\mathrm{N}_{222(12)}\right] \mathrm{NTf}_{2}$. The difference in the density of $\left[\mathrm{P}_{222(12)}\right] \mathrm{NTf}_{2}$ and $\left[\mathrm{N}_{222(12)}\right] \mathrm{NTf}_{2}$ rise to $1.55 \%$. In spite of the larger atomic mass of $\mathrm{P}$ atom than $\mathrm{N}$ atom, a lower simulated density is obtained for all QPILs except $\left[\mathrm{P}_{222(12)}\right] \mathrm{NTf}_{2}$ than the corresponding QAILs. Therefore, any difference in densities of QAILs and QPILs can be attributed to the cation core atom size and the corresponding bond lengths. These results confirm the validity of the utilized force field. Simulated densities of QPILs are not lower than QAILs counterpart by more than 
$2 \%$, and therefore, replacement of $\mathrm{N}$ atom by $\mathrm{P}$ atom does not shift the density much consistent with experiment.

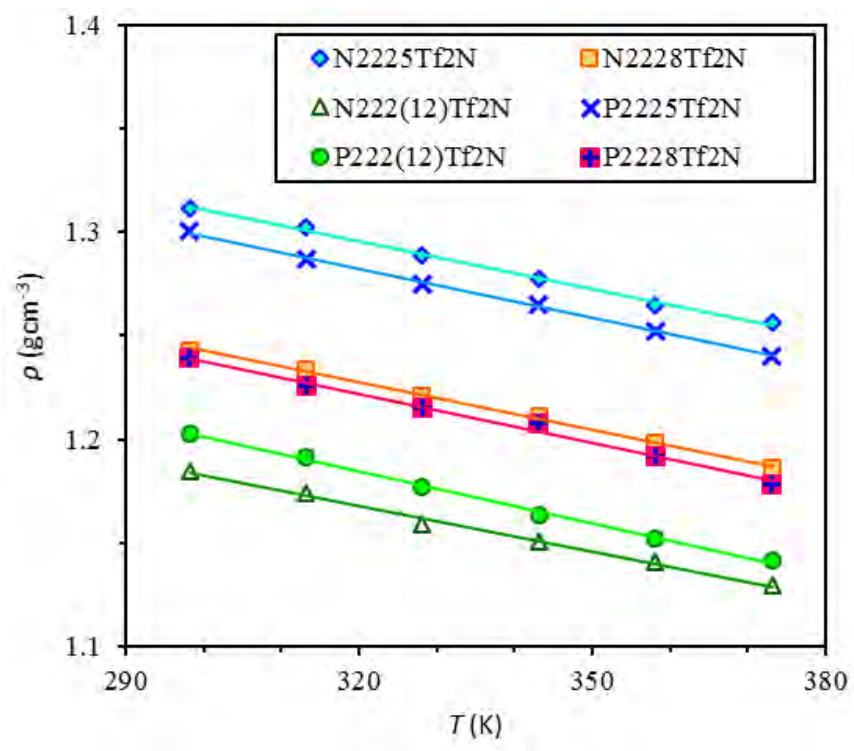

Figure 3. Temperature dependent simulated densities of QAILs and QPILs.

3.2.2 Energy Contributions to the Ensemble. The contributions to the system energy were evaluated by the ensemble utilized in the simulation of density. The van der Waals interaction energy $(E$-vdW) contributing to the total energy ( $E$-tot) of QAILs and QPILs are reported in Table SI13. Values of $E$-vdW (are within -113 to $-179 \mathrm{~kJ} / \mathrm{mol}$ and) increase with temperature and decrease (become more negative) with cation alkyl-chain length in both series of ILs. At all temperatures and alkyl-chain lengths, values of $E$-vdW of QPILs $<$ QAILs, while they are close to one another within $8-10 \mathrm{~kJ} / \mathrm{mol}$.

Contribution of electrostatic energy ( $E$-Coul) to the $E$-tot increases smoothly by increasing temperature in both ILs (Table SI14). Dependence on temperature is small and could be in some part due to computational inaccuracy. At all temperatures and alkyl-chain lengths, the $E$ Coul are all negative and in the order of QPILs $<$ QAILs. Also a turnover is seen in the trend of electrostatic contribution versus chain length: $\left[\mathrm{P}_{2228}\right] \mathrm{NTf}_{2}$ is minimum and $\left[\mathrm{N}_{2228}\right] \mathrm{NTf}_{2}$ is maximum. Considering $E$-Coul is rather high for two types of ILs and knowing QPILs $<$ QAILs with rather high differences (within -17 to $-133 \mathrm{~kJ} / \mathrm{mol}$ ) substantiate that when $\mathrm{N}$ atom is replaced by the $\mathrm{P}$ atom in these ILs, properties will be modified mainly through Coulombic interaction. It worth mentioning that the $E$-vdW is in the range of 8.7 to $16.3 \mathrm{~kJ} / \mathrm{mol}$. 
Liu et al. ${ }^{[66]}$ found a connection between the intermolecular energy (sum of $E$-Coul and $E$ vdW), and the experimental viscosities of QPILs with various alkyl chains. The weaker the ionic interaction the lower the viscosity. However, the lower viscosity of QPILs relative to QAILs cannot be explained by intermolecular energy of the simulated ensemble, since the intermolecular energy of the former is slightly more negative than the latter counterparts. Therefore, this urges elucidating other factor that represents effectively the diffusion ILs with bulky cation when $\mathrm{N}$ atom is replaced by $\mathrm{P}$ atom. Here it is proposed that a cation with lower rotational energy barrier have more accessible conformational states. Then, these accessible states have a consequence on system dynamics, going alongside or substitute for the known free volume mechanism of viscos flow.

3.2.3 Transport Properties. Transport properties are basically originated from the dynamics of constituting atoms and molecules, and mostly studied by self-diffusion and viscosity coefficients. As compared to the organic liquids, low diffusion rate and high viscosity of ILs is attributed to the shape and bulkiness of cation, as well as to the strong cation-anion Coulombic interaction. The partial atomic charges and the van der Waals interactions between alkyl chains substantially influence the dynamics of constituting atoms in the liquid.

Transport properties of the quaternary-based ILs are significantly influenced by the nature of cation core atom. Based on mechanism of particle transport in liquids, diffusion and viscosity may be affected by internal degrees of freedom in particular the conformational rotation of the cation conformers, which crucially must be paid attention when ILs with long alkyl chain are concerned. ${ }^{[\mathbf{8 0 - 8 2}]}$

3.2.4 Self-Diffusion. Coefficients of self-diffusion $(D)$ calculated from atom's trajectories of QAILs and QPILs at $373 \mathrm{~K}$ are listed in Table 3. (Definitions and formulations are given in Supplementary Information, see also Figure SI1 for MSD) In both series of ILs, self-diffusion coefficients of cation $\left(D_{+}\right)$and anion $\left(D_{-}\right)$decreases with alkyl-chain length. Similar to other types of ILs, simulated $D$ values of these ILs strongly depend on the alkyl-chain length. The calculated value of $D_{-}$for $\left[\mathrm{NTf}_{2}\right]^{-}$anion in both series of ILs is larger than those in imidazolium-based ILs. ${ }^{[59]}$ Importantly, based on simulated $D_{+}$and $D_{-}$values, replacement of $\mathrm{N}$ atom of the cation core by $\mathrm{P}$ atom leads to the faster diffusion of both cation and anion (see Table 3).

3.2.5 Viscosity. The advantageous Green-Kubo method is difficult to apply in simulations of highly viscous ILs since the time correlation function converges very slowly. ${ }^{[\mathbf{8 3}-85]}$ Therefore, 
we estimated the viscosity (7) of QAILs and QPILs with reasonable accuracies using StokesEinstein equation,

$$
\eta=\frac{\kappa_{B^{2}}}{-}
$$

where, $k_{n}$ is the Boltzmann constant, $a$ is the molecular diameter, $c$ is a substance dependent parameter, and $D_{i}$ is the sum of cation and anion diffusion coefficient. $c=6$ was adopted for both QAILs and QPILs ${ }^{[86]}$; the value of $a$ for each IL was calculated from simulated liquid density. It is well accepted that a nonpolarizable force field overestimates the viscosity. ${ }^{[\mathbf{6 0}, \mathbf{8 5}]}$ Inclusion of polarization in the force field implicitly (by charge reduction), will improve the simulated dynamic results. ${ }^{[87]}$

First we tried calculation of viscosity by simulation of self-diffusion coefficient for $\left[\mathrm{N}_{2225}\right] \mathrm{NTf}_{2}$ at $373 \mathrm{~K}$ using nonpolarizable force field and original DFT partial atomic charges. After a long production run (14 ns), viscosity with a large deviation (33.28 cP versus experimental value of $10.17 \mathrm{cP}^{[46]}$ ) was obtained. Assuming that this deviation is largely because of lacking polarizability, we included the polarization effect implicitly by using nonpolarizable force field while the original DFT partial atomic charges were scaled down by $10 \% .^{[74]}$

In general, net charges of non-isolated interacting ions of ILs are favourably reduced due to two principal reasons: charge transfer and polarization. Considering the case of polarization, the electron cloud is distorted leading to an effective charge interacting with its neighbours by an apparent reduced charge. A polarizable isolated ion with charge of $1 e$ shall interact with its neighbouring ions like a non-polarizable ion when the net charge is reduced to below $1 e{ }^{[88]}$ So we modeled the effect of polarization by reducing atomic charges up to $10 \%$ uniformly in both ILs types.

Simulated viscosities of QAILs and QPILs at $373 \mathrm{~K}$ with scaled atomic charges are presented in Table 3. The results under scaling are more reliable, where the deviations of simulated viscosity from experiment for QAILs are rather low and increases with alkyl-chain length (within $4.3 \%$ for $\left[\mathrm{N}_{2225}\right] \mathrm{NTf}_{2}$ to $12.4 \%$ for $\left[\mathrm{N}_{222(12)}\right] \mathrm{NTf}_{2}$ ). Also simulated viscosity of QPILs deviates from experimental values ${ }^{[\mathbf{4 3}, \mathbf{4 4}]}$ by $7.4 \%$ for $\left[\mathrm{P}_{2225}\right] \mathrm{NTf}_{2}$ and $19.1 \%$ for $\left[\mathrm{P}_{2228}\right] \mathrm{NTf}_{2}$. Figure 4 demonstrates variation of simulated viscosity with chain length and compares with experimental data.

Simulated viscosity of QPILs are smaller than the QAIL counterparts, in good agreement with experimental observations. ${ }^{[23]}$ These results, meanwhile, qualify the utilized force fields and charge scaling for simulation of QAILs and QPILs. 
As a final remark, the effect of polarizability on density was examined, as in the case of transport properties, at $373 \mathrm{~K}$. The simulated densities were not shifted appreciably as the atomic charges were scaled down.

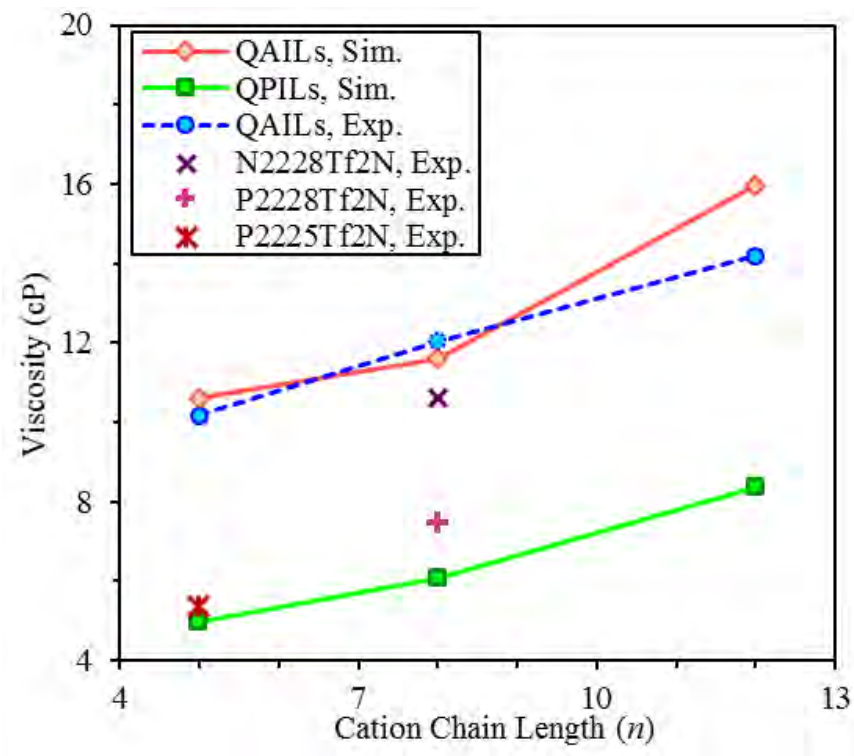

Figure 4. Calculated viscosities of QAILs and QPILs versus cation alkyl-chain length $(n=5,8,12)$ at $373 \mathrm{~K}$. Experimental viscosities for QPILs were extracted from references 43 and 44.

3.2.6 Ionic Conductivity and Transference Number. For both types of ILs, we also calculated the ionic conductivity, which contains information related to ion mobility appreciable in their electrochemical applications. (See Supplementary Information for definitions). Values of molar ionic conductivities at $373 \mathrm{~K}$ are shown in Table 3 . While conductivity decreases with cation alkyl-chain length in both types of ionic liquids, QPILs show substantially higher conductivity than their QAILs counterparts.

The performances and efficiency of these ILs for electrochemical processes can be estimated by transference numbers of cation and anion species $\left(t_{+}\right.$and $\left.t_{-}\right)$. From the calculated transference numbers, shown in Table 3 , it can be seen that $0.40<t_{+}<0.46$ and $0.54<t_{-}<0.6$ for all ILs. While transference number is a measure of contribution of an ion to the current flow, quite importantly, $t_{-}$is larger than 0.5 for anion in all ILs studied, indicating that the electric current is carried by both cation and the anion but chiefly contributed by the anion. The larger transference number of the anion is obviously related to their larger self-diffusion coefficients (see Table 3). Furthermore, it can be concluded that replacement of $\mathrm{N}$ atom of cationic core by $\mathrm{P}$ atom does not alter the relative current flow appreciably. 
Table 3. Simulated self-diffusion coefficients of cation $\left(D_{+}\right)$and anion $\left(D_{-}\right)$(in $\left.10^{-11} \mathrm{~m}^{2} \cdot \mathrm{s}^{-1}\right)$, viscosity $\eta$ (in mPa.s), molar ionic conductivity $\Lambda_{m o l}$ (in $10^{-4} \mathrm{Sm}^{2} \mathrm{~mol}^{-1}$ ) and transference numbers $t$, of QAILs and QPILs at $373 \mathrm{~K}$ with charges scaled by $10 \%$. The molecular diameters $a$ are also shown.

\begin{tabular}{lllllllll}
\hline Ionic Liquid & $\boldsymbol{a}(\AA)$ & $\boldsymbol{D}_{+}$ & $\boldsymbol{D}$. & $\boldsymbol{\eta}_{\text {sim. }}$ & $\boldsymbol{\eta}_{\text {exp. }}$ & $\boldsymbol{\Lambda}_{\text {mol }}$ & $\boldsymbol{t}_{+}$ & $\boldsymbol{t}_{-}$ \\
\hline$\left[\mathbf{N}_{\mathbf{2 2 2 5}}\right] \mathbf{N T f _ { 2 }}$ & 8.43 & 1.29 & 1.77 & 10.60 & $10.17^{\mathbf{4 6}}$ & 0.92 & 0.42 & 0.58 \\
{$\left[\mathbf{N}_{\mathbf{2 2 2}}\right] \mathbf{N T f _ { 2 }}$} & 8.83 & 1.24 & 1.43 & 11.61 & $10.62^{\mathbf{4 4}}$ & 0.80 & 0.46 & 0.54 \\
& & & & & $12.05^{\mathbf{4 6}}$ & & & \\
{$\left[\mathbf{N}_{\mathbf{2 2 2}(12)}\right] \mathbf{N T f}$} & 9.31 & 0.74 & 1.10 & 15.96 & $14.20^{\mathbf{4 6}}$ & 0.55 & 0.40 & 0.60 \\
{$\left[\mathbf{P}_{\mathbf{2 2 2 5}}\right] \mathbf{N T f _ { 2 }}$} & 8.57 & 2.92 & 3.48 & 4.98 & $5.38^{\mathbf{4 3}}$ & 1.92 & 0.46 & 0.54 \\
{$\left[\mathbf{P}_{\mathbf{2 2 2 8}}\right] \mathbf{N T f _ { 2 }}$} & 8.97 & 2.00 & 3.02 & 6.06 & $7.49^{\mathbf{4 4}}$ & 1.51 & 0.40 & 0.60 \\
{$\left[\mathbf{P}_{\mathbf{2 2 2}(12)}\right] \mathbf{N T f}$} & 9.43 & 1.39 & 2.08 & 8.36 & & 1.04 & 0.40 & 0.60 \\
\hline
\end{tabular}

3.2.7 Re-orientation Dynamics. The vector reorientation dynamics (VRD) analysis gprovides information about how fast a vector in the system changes its direction. VRD analysis were performed for $\mathrm{X} \rightarrow \mathrm{CT}$ vector $(\mathrm{X}=\mathrm{N}, \mathrm{P}$; CT=alkyl terminal $\mathrm{C}$ atom) of quaternary ammonium and phosphonium cations and $\mathrm{C} \rightarrow \mathrm{C}$ vector of $\left[\mathrm{NTf}_{2}\right]^{-}$anion.

The re-orientation correlation functions $c_{1}(t)$ were evaluated to describe the re-orientation dynamic behavior of the cations and anions in QAILs and QPILs. $c_{1}(t)$ is simply defined as

$c_{1}(t)=\left\langle\frac{1}{N} \sum_{i=1}^{N} e_{i}(t) \cdot e_{i}(0)\right\rangle$

where $t$ is time, $N$ is number of ionic species and $\boldsymbol{e}_{i}$ stands for a unit vector corresponding to the $\mathrm{X} \rightarrow \mathrm{CT}$ vectors on a cation $i$, and the $\mathrm{C} \rightarrow \mathrm{C}$ vectors on an anion $i$. We found that $c_{1}(t)$ are welldescribed by a stretched exponential decay function for $\mathrm{X} \rightarrow \mathrm{CT}$ of $\left[\mathrm{X}_{222 n}\right] \mathrm{NTf}_{2} \operatorname{ILs}_{\text {where }} n=8,12$.

$f(t)=\exp \left[-\left(\frac{t}{\tau}\right)^{p}\right]$

where $\beta$ is the stretched exponent and $\tau$ is the characteristic correlation time. The average correlation time $\tau_{\sigma}$ is then given by

$\tau_{e}=\int_{0}^{\infty} f(t) d t$ 
Figure 5 Shows $c_{1}(t)$ for the cations and anions of QAILs and QPILs. The values of $\boldsymbol{\rho}$, characteristic re-orientational (correlation) time $\tau$ and average re-orientational (correlation) time $\tau_{\sigma}$ are listed in Table 4.

Calculated $\beta$ values are smaller than unity for both types of ILs, indicating a glassy reorientational motion of cationic alkyl side-chain. QAILs show larger values of both characteristic re-orientational time $\tau$ and average re-orientational time $\tau_{r}$. As a result, reorientational motion of alkyl chain in QPILs is faster than QAILs that could facilitate the translational motion, where the shorter chains move faster than long chains. In a sense, it can be concluded that the relative cation-anion translational motion is always out of phase. And if they form micro-domains, a micro-energetics coexisting domain is involved. Importantly, the present work provides information for ILs involving the complex anion $\left[\mathrm{NTf}_{2}\right]^{-}$, in contrast to simple monoatomic anion like $\mathrm{Cl}^{-[55]}$.
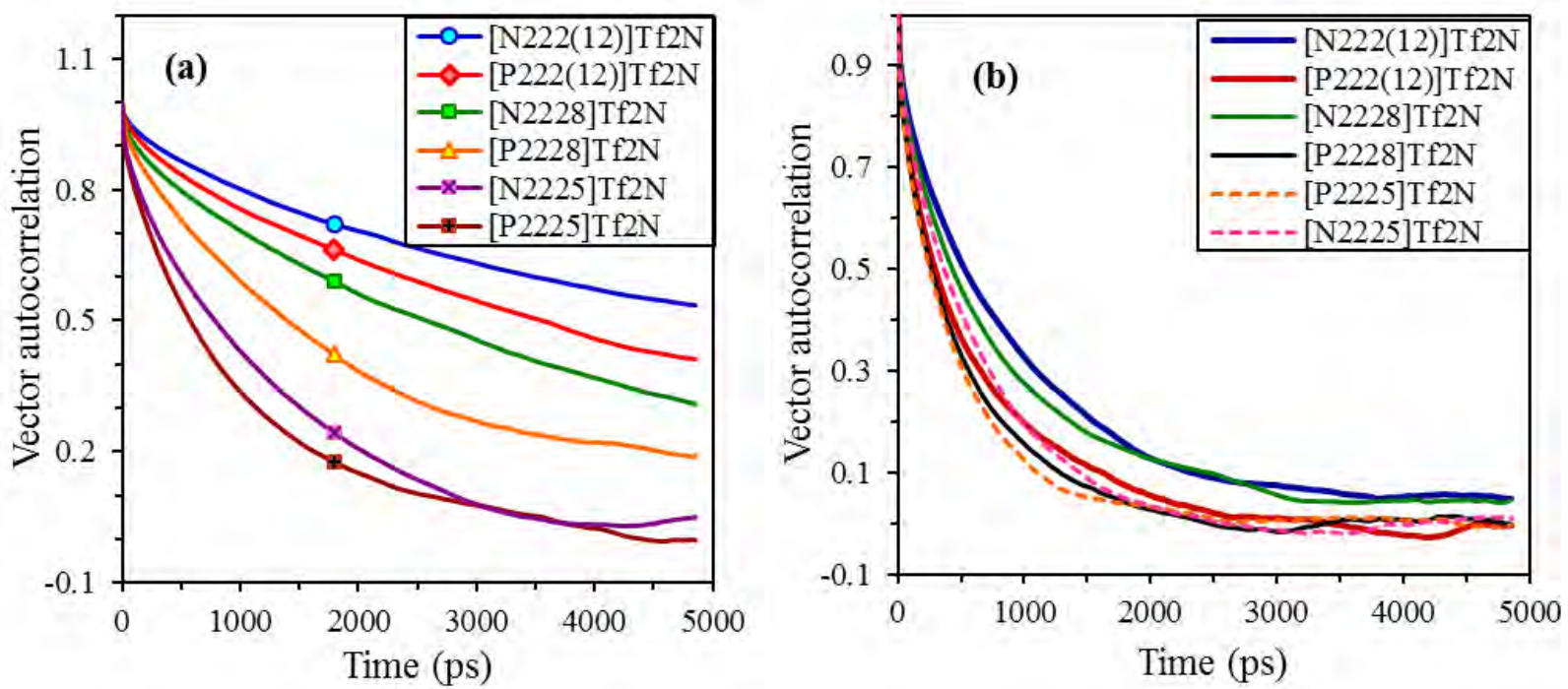

Figure 5. Vector re-orientation dynamics of QAILs and QPILs at 373K: (a) for $X \rightarrow C T(X=N, P)$ vector of cations, (b) for $\mathrm{C} \rightarrow \mathrm{C}$ vector of $\mathrm{NTf}_{2}$ anion.

Table 4. The stretched exponent $\beta$, characteristic re-orientation time $\tau$, and average re-orientation time $\tau_{\varepsilon}$ of the stretched exponential decay function used to fit the re-orientational correlation functions for $\mathrm{X} \rightarrow \mathrm{CT}$ vector on the cation and $\mathrm{C} \rightarrow \mathrm{C}$ vector on the anion for both type of ILs at $373 \mathrm{~K}$.

\begin{tabular}{|l|c|c|c|}
\hline \multirow{2}{*}{ Ionic Liquids } & \multicolumn{3}{|c|}{ Cation $\mathrm{X} \rightarrow \mathrm{CT}$ vector } \\
\cline { 2 - 4 } & $\beta$ & $\tau / \mathrm{ns}$ & $\tau_{\varepsilon} / \mathrm{ns}$ \\
\hline$\left[\mathrm{N}_{2228}\right] \mathrm{NTf}_{2}$ & 0.7710 & 4.03 & 2.66 \\
\hline
\end{tabular}




\begin{tabular}{|l|c|c|c|}
\hline$\left[\mathrm{N}_{222(12)}\right] \mathrm{NTf}_{2}$ & 0.6600 & 9.77 & 3.37 \\
\hline$\left[\mathrm{P}_{2228}\right] \mathrm{NTf}_{2}$ & 0.7034 & 2.21 & 1.95 \\
\hline$\left[\mathrm{P}_{222(12)}\right] \mathrm{NTf}_{2}$ & 0.7526 & 5.70 & 3.01 \\
\hline
\end{tabular}

3.2.8 Ion-Pair Lifetime Ion-pair (IP) correlation function was calculated using equation SI5. ${ }^{[89-91]}$ Our calculations in the first place indicate that QAILs have larger ion-pair lifetime than QPILs (see Table SI15). In the second place, at low temperature (298K, Figure 6(a)), as the cation alkyl-chain length increases, the ion-pair lifetime of QAILs and QPILs become closer to each other whereas at high temperature (373K, Figure 6(b)), the ion-pair lifetime of both series become farther apart from each other. This indicates that at low temperature the lifetime is determined by the influence of structure, while at high temperature the thermal energy determines the lifetime. In any case the lifetime could be an indication of the frictional forces of the ion-pair when the molecular motion in a fluid is concerned. The higher the lifetime the higher the contribution to frictional force. Hence, the lifetime is in the order of QPILs $<$ QAILs consistent with the order of their viscosities.
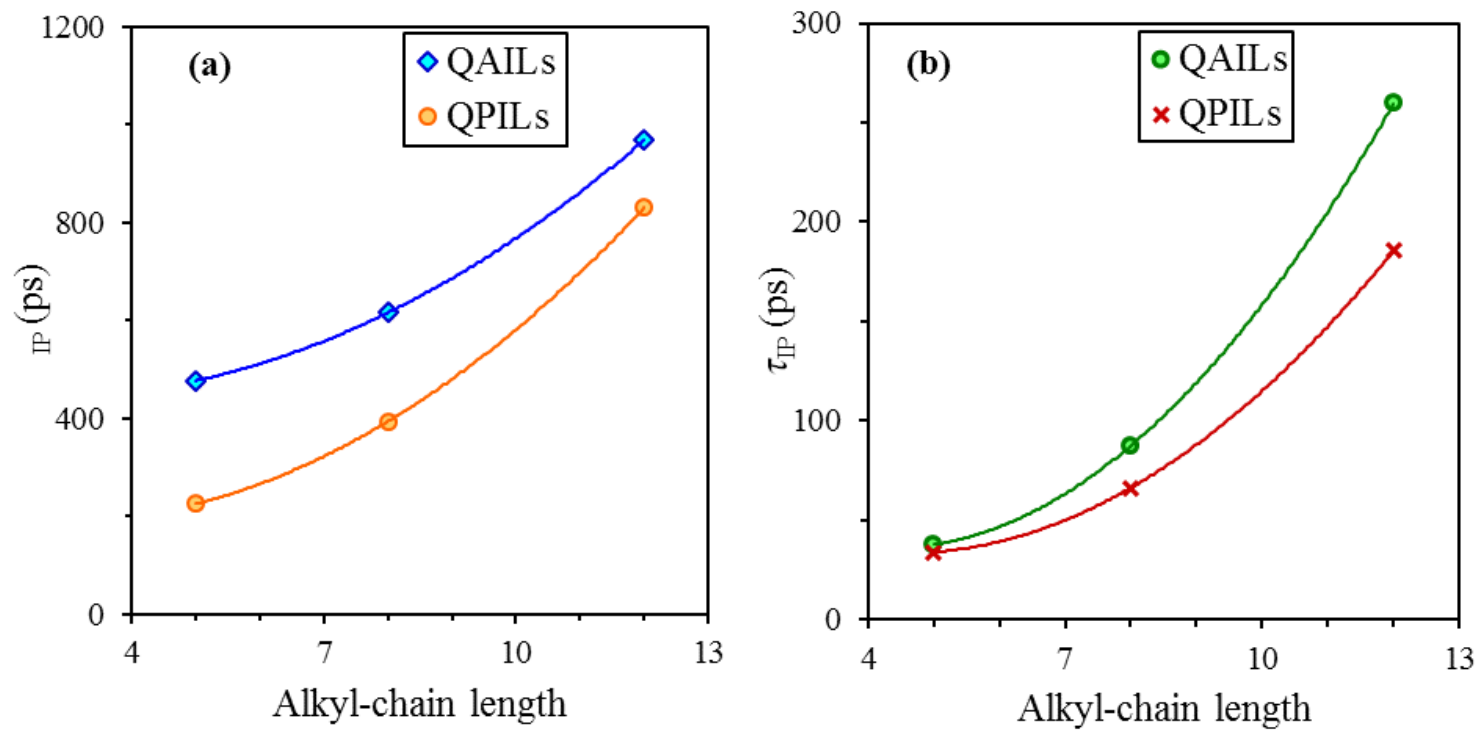

Figure 6. Calculated ion-pair lifetime of QAILs and QPILs (a) at $298 \mathrm{~K}$ and (b) at $373 \mathrm{~K}$.

To further elaborate on the cation-anion association, we compute the average number of anions neighboring each cation within the first solvation shell, as shown in Table SI16. At $373 \mathrm{~K}$, cations in $\left[\mathrm{N}_{222(12)}\right] \mathrm{NTf}_{2}$ and $\left[\mathrm{P}_{222(12)}\right] \mathrm{NTf}_{2}$ have the minimum association with anion, $n\left[\mathrm{X}_{222(12)}\right]\left(\mathrm{NTf}_{2}\right)=5$, and $\left[\mathrm{N}_{2225}\right] \mathrm{NTf}_{2}$ and $\left[\mathrm{P}_{2225}\right] \mathrm{NTf}_{2}$ show the maximum association, $n\left[\mathrm{X}_{2225}\right]\left(\mathrm{NTf}_{2}\right)=7$ (where X: N, P). (see 
Table SI16). In general, the anion-cation lifetime of the QPILs at maximum neighboring number is lower than QAILs, which again confirm the order of their viscosities.

\subsection{Liquid Bulk Structure}

3.4.1 Radial Distribution Functions. Most unique properties shown by ILs originate from the anion-cation interaction and their relative structure. Valuable microscopic information can be gained by contrasting structural relationship, as we did by calculation of various site-site radial distribution functions (RDFs) over $4 \mathrm{~ns}$ trajectories in the temperature range 298 to 373 $\mathrm{K}$ and 1 atm pressure for QAILs and QPILs.

RDF Analysis indicates that the correlation between the alkyl terminal carbon atoms (CT) of QAILs and QPILs is the strongest compared with other chain atoms. Though a detail consideration (Figure 7) indicates that the tail including $\mathrm{C}_{12}-\mathrm{C}_{11}-\mathrm{C}_{10}$ atoms could have appreciable side-by-side correlation. (see Figure 7 for $\left[\mathrm{N}_{222(12)}\right] \mathrm{NTf}_{2}$ )

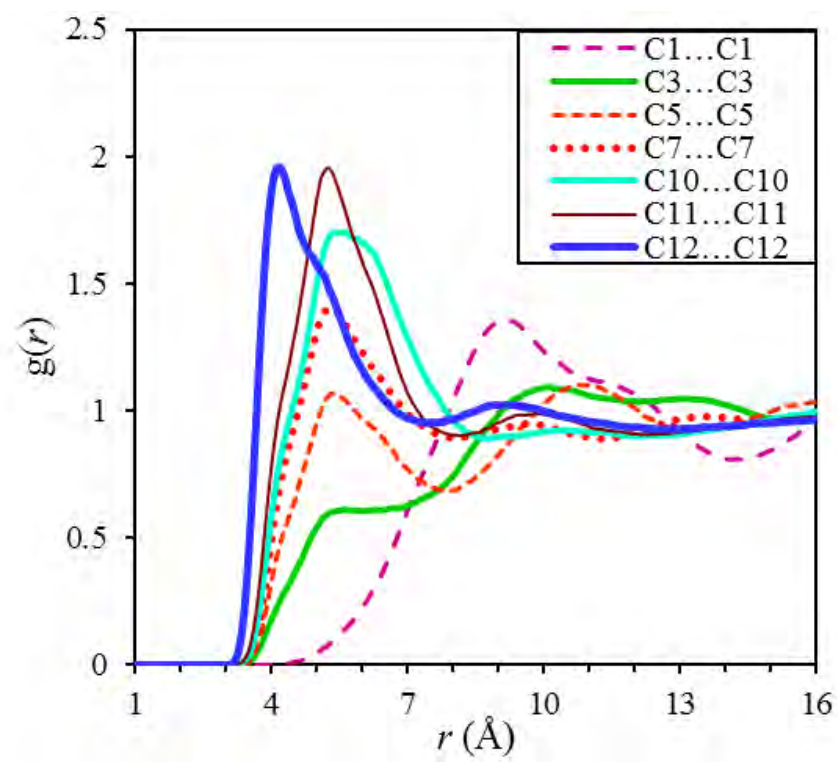

Figure 7. Site-site intermolecular radial distribution function of terminal carbon atom (CT) with carbon atoms of alkyl-chain in $\left[\mathrm{N}_{222(12)}\right] \mathrm{NTf}_{2} \mathrm{IL}$.

A strong and a well-structured CT...CT correlation finds generality in all QAILs and QPILs as can be verified in Figures 8(a) and 8(b). In both series, a sharp peak is seen around 4 $\AA$ with the distance of closest approach of $3.25 \AA$, in good agreement with the literature. ${ }^{[61]} \mathrm{We}$ compare the peak positions under the condition that distribution function plots have resolution of $0.05 \AA$ and the uncertainty of the calculation are the same in all simulations. Position of first 
peak shifts to higher distances (from 2.04 to $2.14 \AA$ for QAILs and from 1.77 to $2.17 \AA$ ) as the cation alkyl-chain length increases. In each series, the intensity of main peak increases with alkyl-chain length of cation. (However, in QAILs this peak is slightly higher than QPILs and the peak height changes with chain length with a higher rate in the latter ILs than the formers.) Our results are in agreement with the wok of Scarbath-Evers et al. ${ }^{[55]}$ Similar trend has been reported for imidazolium-based ionic liquids, ${ }^{[\mathbf{9 2 9}, 93]}$ and $\left[\mathrm{N}_{n n n(1)}\right] \mathrm{NTf}_{2} \mathrm{ILs}^{[61]}$ indicating that the tendency of segregated nonpolar domains formation is increased as the size of the nonpolar moieties of molecule (the alkyl-chain) becomes larger (Figure 8). That the RDFs peak intensities and viscosities are increased with the alkyl-chain length imply a direct correlation. Particularly, CT $\cdots$ CT RDF intensity and the viscosity in both series of ILs are correlated.
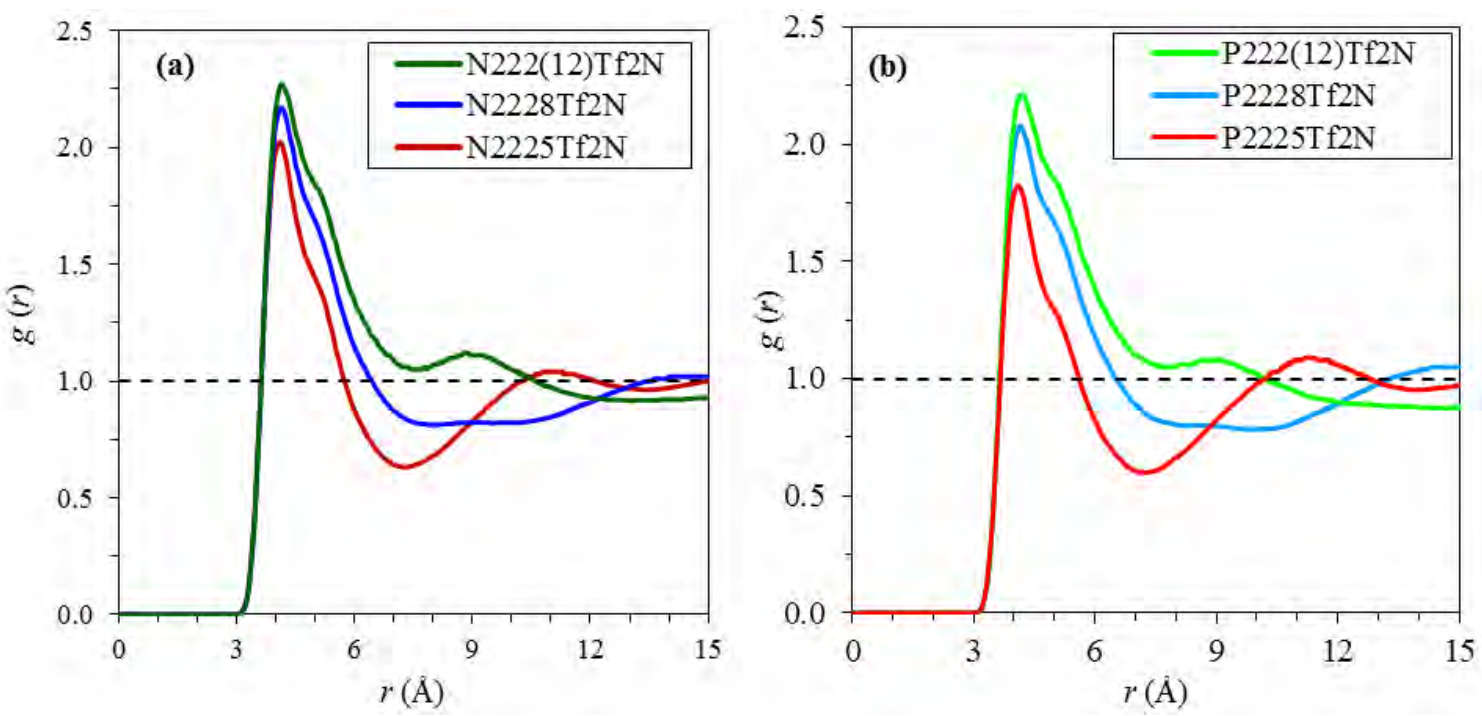

Figure 8. Simulated CT...CT correlation functions for (a) $\left[\mathrm{N}_{222 n}\right] \mathrm{NTf}_{2}$ and (b) $\left[\mathrm{P}_{222 n}\right] \mathrm{NTf}_{2} \operatorname{ILs},(n=5,8,12)$ at $373 \mathrm{~K}$.

To assess the cation-anion correlation, we selected $\mathrm{N} \cdots \mathrm{O}$ and $\mathrm{P} \cdots \mathrm{O}$ RDFs in QAILs and QPILs, respectively (Figure 9). A strong first peak occurs as followings: $\left[\mathrm{N}_{2225}\right] \mathrm{NTf}_{2}, 4.55 \AA$; $\left[\mathrm{N}_{2228}\right] \mathrm{NTf}_{2}$, $4.62 \AA ;\left[\mathrm{N}_{222(12)}\right] \mathrm{NTf}_{2}, 4.62 \AA ;\left[\mathrm{P}_{2225}\right] \mathrm{NTf}_{2}, 4.76 \AA$; $\left[\mathrm{P}_{2228}\right] \mathrm{NTf}_{2}, 4.71 \AA ;$ [ $\left.\mathrm{P}_{222(12)}\right] \mathrm{NTf}_{2}, 4.79 \AA . \mathrm{A}$ second less intense and broader peak are seen around 7.0 (QPILs) and $6.9 \AA$ (QAILs). The intensity of first peak increases appreciably with the cation size. This represents the fact that the correlation between ionic species becomes stronger as the cation alkyl-chain length increases, which can be attributed to a high alkyl-alkyl organization. As a result, the anion-cation structural relation in QPILs is weaker and of higher dynamic than QAILs (compare Figure 9(a) with 9(b)). This will be studied further in detail by spatial distribution functions. 

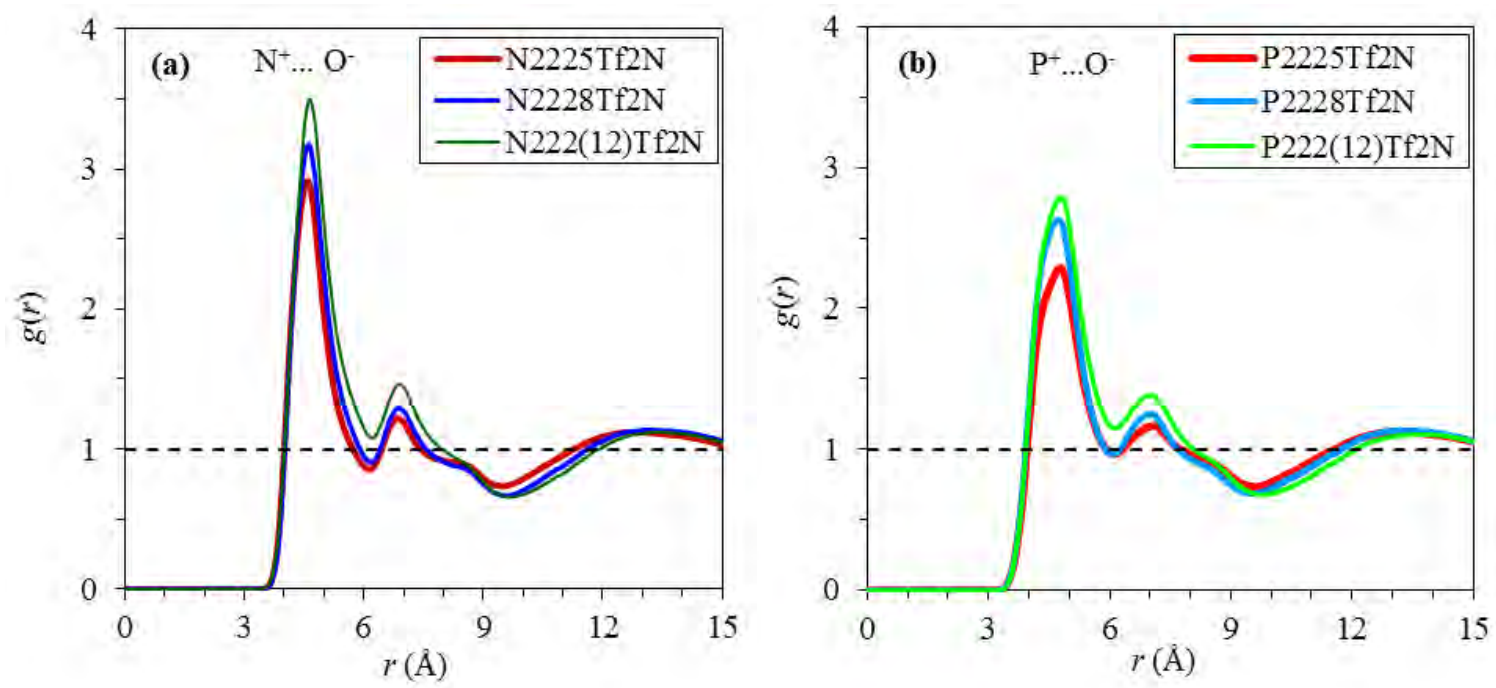

Figure 9. Cation-anion RDFs for QAILs and QPILs atoms at $373 \mathrm{~K}$ for, (a) N (cation) to O (anion) and (b) P (cation) to $\mathrm{O}$ (anion).

As already mentioned, QAILs show higher viscosities than their QPILs counterparts. Consistent with this, the intensity of first peak in RDF of the former ILs is higher than the latter's. For a qualitative topology analysis, the polar and nonploar domains are visualized by color coding of a snapshots of the simulation box as shown in Figure SI2. The negative part includes all atoms of the anion; the positive part contains cation atoms including $\mathrm{N}$ atom, seven carbon atoms ( $\mathrm{C} 1$ and $\mathrm{C} 2$ bonded to $\mathrm{N}$ ), and seventeen hydrogen atoms ( $\mathrm{H} 1$ and $\mathrm{H} 2$ bonded to $\mathrm{C} 1$ and $\mathrm{C} 2$ ); the nonpolar domain includes all atoms of the alkyl-chain except $\mathrm{C} 1$ and $\mathrm{H} 1$. As seen in Figure SI2, for short alkyl-chains ( $\left[\mathrm{N}_{2225}\right] \mathrm{NTf}_{2}$ and $\left.\left[\mathrm{P}_{2225}\right] \mathrm{NTf}_{2}\right)$, the nonpolar domains consist of hydrocarbon-like islands in the midst of a continuous polar network, whereas for longer alkyl-chains those islands start to merge forming a second continuous micro-phase, establishing in this way a bi-continuous segregated phase.

In this regards, our simulation results are in good consistency with findings of Griffin $e t$ al. $^{[49]}$ They indicated that, by both structural and dynamical studies, QAIL and QPIL exhibit nano-phase segregation of the charge-rich ionic centers from the non-polar alkyl-chains. Their results suggest that the alkyl-rich nano-domains of $\left[\mathrm{N}_{2228}\right] \mathrm{NTf}_{2}$ are slightly larger and more well-defined than those of $\left[\mathrm{P}_{2228}\right] \mathrm{NTf}_{2}$. They have compared and attributed the contrast in local dynamics of $\left[\mathrm{N}_{2228}\right] \mathrm{NTf}_{2}$ and $\left[\mathrm{P}_{2228}\right] \mathrm{NTf}_{2}$ to a direct result of differences in the interionic interactions in these seemingly similar ILs. ${ }^{[49]}$ However, gas phase results of Scarbath-Evers et al. ${ }^{[55]}$ and the present work indicate that difference in interionic interaction and the intermolecular energy (of the ensemble) comparatively do 
not account for the order of dynamic properties like viscosity and ion conductivity of QAIL and QPIL correctly.

3.4.2 Nano-structure Domain Analysis. To achieve more qualitative and quantitative insight into the effect of cation core atom on shape and size of ILs nano-structures we used a newly developed domain analysis tool implemented in TRAVIS program. ${ }^{[94]}$ The domains are built up with the aid of radical Voronoi tessellation. ${ }^{[95]}$ The atoms of a molecular subset provide the Voronoi sites with particular Voronoi radii such as van der Waals radii. This results in useful average quantities, such as domain volume and surface, as well as the isoperimetric quotient which indicates deviation from a spherical shape.

If the domain has the shape of a sphere, the isoperimetric quotient will be 1 ; otherwise, it will be smaller than 1. Deviation from unity describes the asphericity of the domain.

Table 5. Nano-domain analysis of QAILs and QPILs at 373K, 10\% reduced charge.

\begin{tabular}{|l|l|l|l|c|c|}
\hline Ionic Liquids & Domain & Domain count & $\begin{array}{c}\text { Volume } \\
\left(\AA^{3}\right)\end{array}$ & $\begin{array}{c}\text { Surface area } \\
\left(\AA^{2}\right)\end{array}$ & $\begin{array}{c}\text { Isoperimetric } \\
\text { Quotient }\end{array}$ \\
\hline$\left[\mathrm{N}_{2225}\right] \mathrm{NTf}_{2}$ & $\begin{array}{l}\text { polar } \\
\text { nonpolar }\end{array}$ & 1.0 & 72022 & 19365 & 0.28 \\
\hline$\left[\mathrm{N}_{2228}\right] \mathrm{NTf}_{2}$ & polar & 1.0 & 1872 & 1882 & 0.54 \\
\hline$\left[\mathrm{N}_{222(12)}\right] \mathrm{NTf}_{2}$ & nonpolar & 1.5 & 71668 & 24205 & 0.20 \\
& nolar & 1.0 & 71916 & 28271 & 0.20 \\
\hline$\left[\mathrm{P}_{2225}\right] \mathrm{NTf}_{2}$ & polar & 1.0 & 75827 & 19445 & 0.16 \\
& nonpolar & 13.1 & 1645 & 1625 & 0.53 \\
\hline$\left[\mathrm{P}_{2228}\right] \mathrm{NTf}_{2}$ & polar & 1.0 & 76057 & 24792 & 0.21 \\
& nonpolar & 1.7 & 23715 & 17863 & 0.24 \\
\hline$\left[\mathrm{P}_{222(12)}\right] \mathrm{NTf}_{2}$ & polar & 1.0 & 75836 & 28561 & 0.17 \\
& nonpolar & 1.0 & 50551 & 28538 & 0.11 \\
\hline
\end{tabular}

According to domain analysis reported in Table 5, $\left[\mathrm{N}_{2225}\right] \mathrm{NTf}_{2}$ and $\left[\mathrm{N}_{2228}\right] \mathrm{NTf}_{2}$ ILs show larger nonpolar volume domain than their phosphonium counterparts. This finding is in good agreement with the results of Griffin et al. ${ }^{[49]}$

Results of Table 5 show that, domain count is larger for phosphonium ILs with $n=5$ and 8 . Surprisingly, the alkyl chain length has a profound effect of the domain count of both ILs series. In both series, the nonpolar domain become more continuous by increasing cation alkyl chain, such that for $n=12$ the liquid system merges into only two separated continuous domains. Therefore, in comparison to QPILs, shorter and medium alkyl side chains in QAILs can aggregate more freely and form a larger nonpolar nano-domains. Clearly as shown in Figure 9, these can be attributed to the correlation of anion with cation head group which is stronger for QAILs than QPILs. These 
properties, although static, can be used in the favour of confirming dynamic properties simulated here and those reported experimentally.

The results of Table 5 indicate that both "nature of cation central atom" and "cation alkyl chainlength" are responsible for Nanodomain formation in QAILs and QPILs. As reported in Table 5, the difference in nonpolar domain count of QAILs and QPILs decreases by increasing cation alkyl-chain length. In shorter alkyl chains $(n=5)$, nonpolar domain count is more affected by nature of cation central atom, where in larger alkyl chains $(n=12)$, nonpolar domain count is more affected by cation alkyl chain-length. In short and medium alkyl chains $(n=5,8)$ larger nonpolar domain count (smaller size nonpolar domains) of QPILs can be attributed to the weaker correlation of anion with cation head group in comparison with QAILs.

Also by increasing cation alkyl-chain length, the difference in nonpolar domain count of QAILs and QPILs seemingly vanishes, which shows prominent effect of the cation alkyl-chain length.

3.4.3 Combined Radial/Angular Distribution Function. From the combined radial/angular distribution function shown in Figure 10, it can be concluded that the positioning of the anion with respect to the cation in both QAILs and QPILs defined by angle and distance are generally the same. Any positional rearrangement goes along with an angular readjustment almost similarly in both type of ILs. However, anion in QPILs spans more widely both positionally and spatially in the domain of $60^{\circ}-70^{\circ}, 80^{\circ}-105^{\circ}, 160^{\circ}-180^{\circ}$, a clear indication of more anion-cation relative dynamics with respect to that of QAILs. Besides, the domain in the range of $80^{\circ}-90^{\circ}$ shows more enhancements for QPILs than QAILs and represents additional dynamics of anion with respect to cation for the former ILs. The quantitative analysis discussed here for the case of $n=8$ is applied similarly to the cases $n=5$ and 12 . 


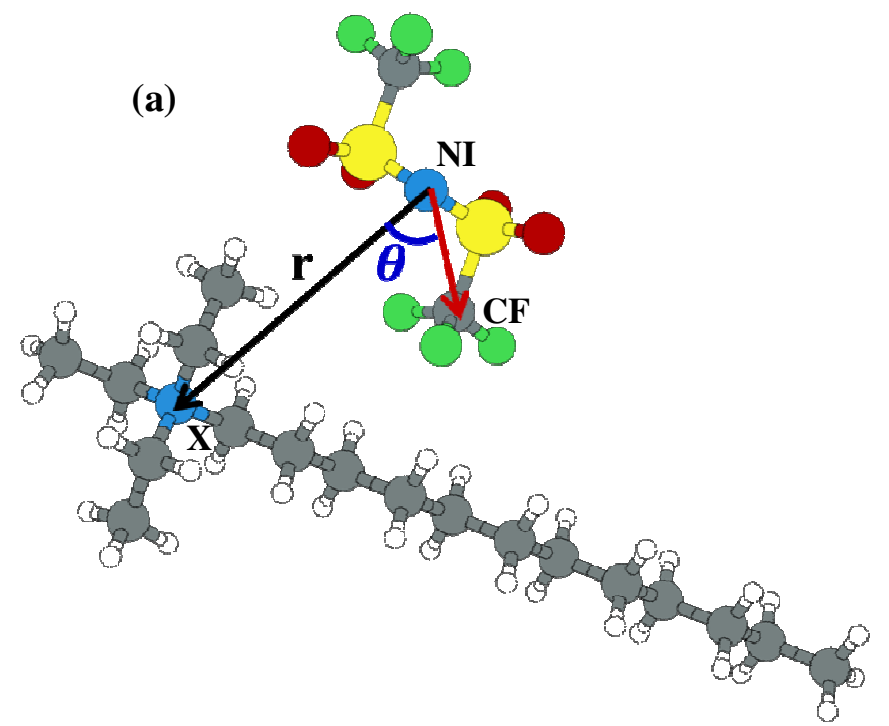

(b) $\quad\left[\mathrm{N}_{2228}\right] \mathrm{Tf}_{2} \mathrm{~N}$

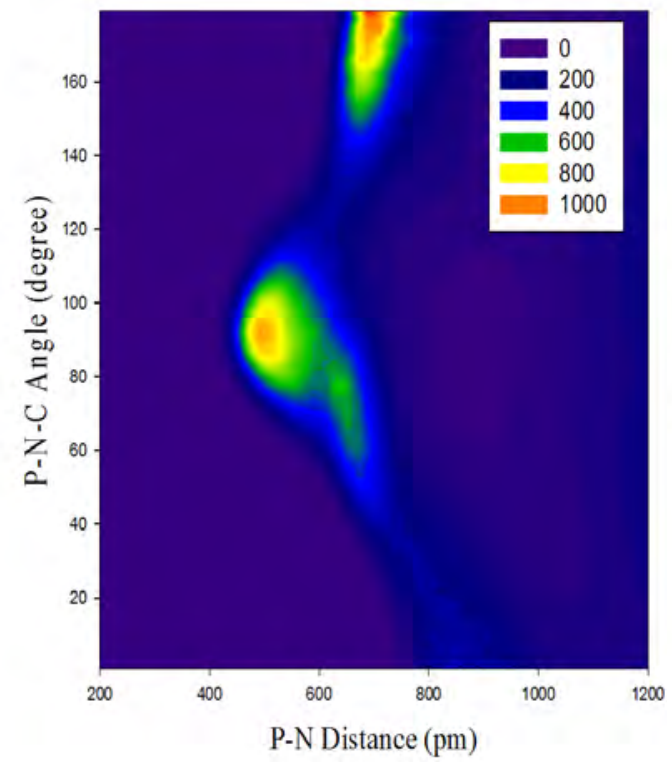

(c) $\left[\mathrm{P}_{2228}\right] \mathrm{Tf}_{2} \mathrm{~N}$

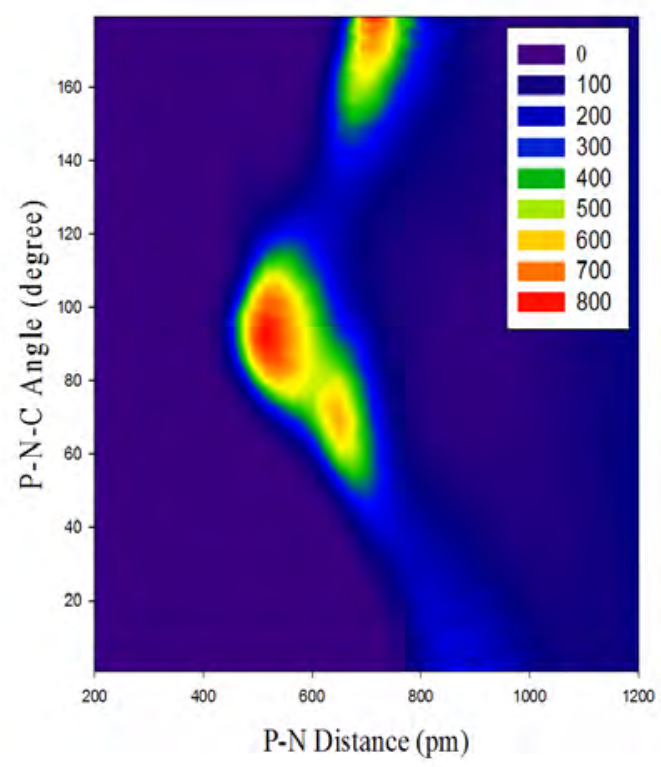

Figure 10. (a) Vector definition $(\mathrm{NI} \rightarrow \mathrm{X}(\mathrm{X}: \mathrm{N}, \mathrm{P})$ and $\mathrm{NI} \rightarrow \mathrm{CF})$ for Combined radial/angular distribution analysis; (b) and (c) combined radial/angular distribution functions between $\left[\mathrm{X}_{2228}\right]^{+}$and $\mathrm{NTf}_{2}{ }^{-}(\mathrm{X}=\mathrm{N}, \mathrm{P})$, see also Figure SI3 for the cases $n=5,12$.

3.4.4 Conformational Analysis by Dihedral Angles Distributions. The distributions of dihedral angles $\left(\mathrm{C}^{4}-\mathrm{C}^{3}-\mathrm{X}-\mathrm{C}^{1} ; \mathrm{X}: \mathrm{N}, \mathrm{P}\right)$ were obtained in the equilibrated ensembles containing 150 ion-pairs of (each type of) ILs at $298 \mathrm{~K}$. Two points can be deduced from the results shown in Figure 11: (1) domain and diversity, (2) the peak frequency values. A lower peak frequency (e.g., occurrence) and a higher diversity is the characteristics of $\left[\mathrm{P}_{2225}\right] \mathrm{NTf}_{2}$, while $\left[\mathrm{N}_{2225}\right] \mathrm{NTf}_{2}$ has the feature of higher peak frequency and lower diversity. All these can be 
explained by lower rotational energy barrier of the former, which accounts microscopically for the lower transport coefficient (Figure 2). As demonstrated in Figure 11(a), the number of $\left[\mathrm{N}_{2225}\right] \mathrm{NTf}_{2}$ ion-pairs with lower dihedral angles is more than ion-pairs having higher dihedral angles.

In the case of $\left[\mathrm{N}_{2225}\right] \mathrm{NTf}_{2} \mathrm{IL}$, all the dihedral states are distributed over the range $0^{\circ}$ to $180^{\circ}$, showing two separate domains concentrated around the low angle $\tau \approx 50^{\circ}-60^{\circ}$ and the high angle $\tau \approx 160^{\circ}-170^{\circ}$ are more probable. This is the case for $\left[\mathrm{P}_{2225}\right] \mathrm{NTf}_{2}$ except that the domains are not separated strictly.

The occurrence of dihedral angles for each ILs of both series is compared in Figure SI4. Dihedrals tend very much similar in both occurrence and angles at high alkyl chain length. However, at low and mid chain length, differences are evidence showing a shift in angle and occurrence (for $n=5$ ) and only a shift in occurrence for the case of $n=8$.
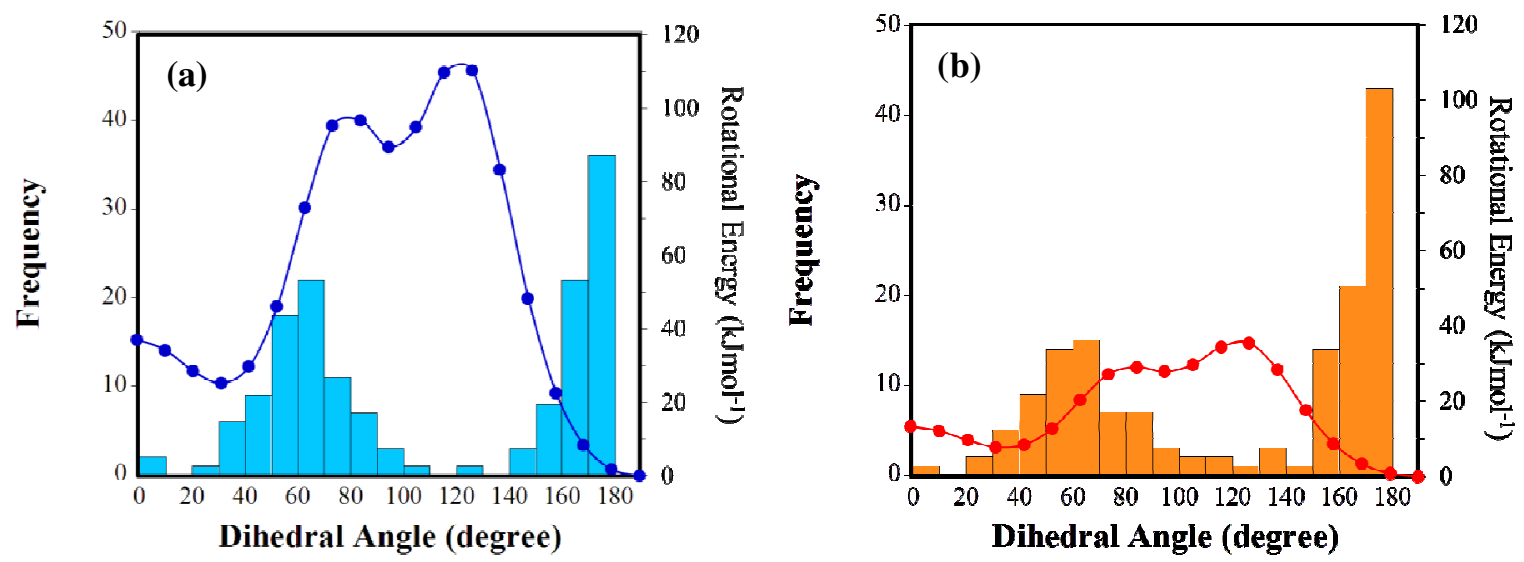

Figure 11. The distribution of dihedral angles in ensembles containing 150 ion-pairs of ILs (a) $\left[\mathrm{N}_{2225}\right] \mathrm{NTf}_{2}$ and (b) $\left[\mathrm{P}_{2225}\right] \mathrm{NTf}_{2}$ at $298 \mathrm{~K}$. Bars are the frequency. Dots are rotational energy barriers calculated at MP2/6-311++G(d,p) level of theory (Figure 2) and adopted here.

3.4.5 Spatial Distribution Functions. Spatial distribution function (SDF), which is the projection of three-dimensional distribution of atom density around a given atom or group of atoms, was used to extract useful visual structural information from MD simulations. calculated by TRAVIS package ${ }^{[93]}$ in particular SDFs of $\left[\mathrm{NTf}_{2}\right]^{-}$anion around head groups of quaternary ammonium and phosphonium cations are shown in Figure SI5 to verify the deviation in properties from the differences in their anion-cation correlation. Figures. SI6(a) to SI6(f) illustrate the probability of finding head group of cation around head group of a given cation in both QAILS and QPILs. The SDFs analysis demonstrates the first and second ionic shells around the $\left[\mathrm{N}_{222 n}\right]^{+}$(or $\left[\mathrm{P}_{222 n}\right]^{+}$) cations has a spherical character, centered at $\mathrm{N}$ (or $\mathrm{P}$ ) atom. For shorter alkyl-chain lengths, the SDF tends complete spherical shape. However, the 
spherical SDFs are ruptured by larger cations with long alkyl-chain length (e.g., $n=12$ ) protruding outward with an angle with respect to cation located at the sphere center.

Comparing anion-cation SDFs (Figure SI5) unravels that the probability of finding anion around cation head group in QAILs is larger than QPILs, confirming the stronger cation-anion correlation in QAILs, hence their higher viscosity. The increase in cation alkyl chain length breaks the spherical distribution of anion around cation. In good consistency with findings of Griffin et $a l .^{[49]}$, the anions are associated much more readily with the cation head group of QAILs than to QPILs. A more well-defined exclusion of anions from the alkyl-rich nanodomains of QAILs occurs that results in larger non-polar nano-domains.

On the other hand, the cation-cation SDFs in QAILs are more adhered to spherical shape than in QPILs, as demonstrated in Figure SI6. The rupture in spherical distribution of cation around central cation is more pronounced in QPILs than in QAILs. These findings confirm the differences in bulk microstructures of these two types of ILs, and therefore the difference in their physical properties, particularly the dynamics properties.

From calculated averaged anion density around cation head group we concluded that the probability for anion around cation in QAILs is larger than QPILs. (see Table 6)

Table 6. Calculated averaged anion density around cation head group.

\begin{tabular}{|c|c|c|c|}
\hline QAILs & $\begin{array}{c}\text { averaged anion density } \\
\left(\mathrm{nm}^{-3}\right)\end{array}$ & QPILs & $\begin{array}{c}\text { averaged anion density } \\
\left(\mathrm{nm}^{-3}\right)\end{array}$ \\
\hline$\left[\mathrm{N}_{2225}\right] \mathrm{NTf}_{2}$ & 24.9517 & {$\left[\mathrm{P}_{2225}\right] \mathrm{NTf}_{2}$} & 23.6828 \\
\hline$\left[\mathrm{N}_{2228}\right] \mathrm{NTf}_{2}$ & 21.3834 & {$\left[\mathrm{P}_{2228}\right] \mathrm{NTf}_{2}$} & 20.5077 \\
\hline$\left[\mathrm{N}_{222(12)}\right] \mathrm{NTf}_{2}$ & 18.3835 & {$\left[\mathrm{P}_{222(12)}\right] \mathrm{NTf}_{2}$} & 17.8469 \\
\hline
\end{tabular}

\section{CONCLUSIONS}

Nature of central core atom of cation influences the structure, dynamic and thermophysical properties of two classes of ionic liquids, QAILs and QPILs. The differences in their properties related to electronic structure like bond length, partial charge distribution, were all accessed by QCC. A direct access to dynamics responsible for the appreciable difference in transport properties was achieved by calculation of potential energy changes during rotation about the $\mathrm{N}-\mathrm{C}$ and P-C single bond of the two types of ILs. Thanks to $\left[\mathrm{NTf}_{2}\right]^{-}$anion which involves smooth anion-cation interaction (contrary to say $\mathrm{Cl}^{-}$anion) and thus enabled rather accurate simulation of transport properties favorable for differentiating the features of the two series of ILs. 
Ammoniums are generally found to possess slightly denser liquid structure than phosphoniums. While, the polarization should be adjusted efficiently to enable accurate simulation of transport properties, simulated densities are insensitive to scaling down the atomic charges, and hence to polarization effects. Therefore, a small change in liquid structure brings about high alteration in liquid transports magnitudes as studied by diffusion and viscosity. It looks that for such systems with complex molecular structure, the internal degrees of freedom are essential in determination of liquid dynamic properties. The simulated viscosity of phosphoniums is appreciably smaller than the ammoniums, what makes the former ILs more striking over the latter for higher electrochemical performances. The $\left[\mathrm{NTf}_{2}\right]^{-}$anion that is found to be the major electric charge carrier has essentially the same transference number in both types of ILs. Significantly, the accuracy of simulated transport properties (self-diffusion coefficients, viscosities, molar conductivities and transference numbers) is enhanced (in particular for the viscosity) by scaling down the atomic charges. These observations also consistent in a general way with the results of perturbation theory of the dense fluid (in statistical mechanics) that the liquid structure is determined by the repulsive side of the potential function.

Both types of ILs make nano-scale domains consisting nonpolar alkyl chains. However, nano-aggregates in QPILs are weaker than in QAILs, leading to smaller nano-aggregates for the former. While these are solely studied by simulated RDFs, the SDFs indicate a more nonspherical anion distribution around the cation in QPIL than in QAIL, which is enhanced as the alkyl-chain length increases. As a conclusion, by the virtue of P central cation atom, QPILs are preferred in various applications such as electrochemistry as evidenced by un-altered simulated transference number in addition to having lower viscosity than QAILs.

Analysis of molecular transport dynamics including ion-pair lifetime and vector reorientation as well as the nano-structure domain analysis confirm faster dynamics for cation of phosphoniums than ammoniums. $\left[\mathrm{NTf}_{2}\right]^{-}$anion features a long lived pairing with both types of cations. Ammoniums ionpair have longer life-time (up to a factor of 2.1) than phosphoniums, which can be examined by visual observations.

Replacement of $\mathrm{N}$ atom by $\mathrm{P}$ atom does not alter much the equilibrium thermodynamic properties. This replacement, however, modifies the electronic structural properties and modifies the atomic charge distribution in addition to the increase in length of C-P bond appreciably. As a consequence of such modifications, the rotational energy barrier of phosphoniums conformer is lowered substantially, which explains their substantial lower viscosity compared to ammoniums. More precisely, since the cation alkyl group has a 
profound effect on the viscosity, the idea of free volume as a part of viscos flow mechanism for phosphoniums is fulfilled under the condition that their cations could deform easily due to low molecular friction and stress-free access to more conformational states and hence having reduced viscos forces appreciably.

\section{ACKNOWLEDGEMENTS}

The authors are indebted to the research council of the Shiraz University and National Science Foundation (Grant no. 93044214) for financial supports. Parallel computing facilities are granted in part by the Enhanced Oil Recovery (EOR) Center of the College of Engineering. Also, cluster computing times, provided in part by the High Performance Computing research laboratory of Institute for Research in Fundamental Sciences (IPM), are greatly acknowledged.

\section{REFERENCES}

[1] N. V. Plechkova, K. R. Seddon, Chem. Soc. Rev. 2008, 37, 123-150.

[2] J. S. Wilkes, J. Mol. Catal. A: Chem. 2004, 214, 11-17.

[3] G. T. Wei, Z. Yang, C. Y. Lee, H. Y. Yang, C. R. C. Wang, J. Am. Chem. Soc. 2004, 126, 5036-5037.

[4] H. Itoh, K. Naka, Y. Chujo, J. Am. Chem. Soc. 2004, 126, 3026-3027.

[5] H. Sakaebe, H. Matsumoto, K. Tatsumi, J. Power Sources 2005, 146, 693-697.

[6] H. Matsumoto, H. Sakaebe, K. Tatsumi, M. Kikuta, E. Ishiko, M. Kono, J. Power Sources 2006, $160,1308-1313$.

[7] R. Kawano, H. Matsui, C. Matsuyama, A. Sato, M. A. B. H. Susan, N. Tanabe, M. Watanabe, J. Photochem. Photobiol. A Chem. 2004, 164, 87-92.

[8] S. Mikoshiba, S. Murai, H. Sumino, T. Kado, D. Kosugi, S. Hayase, Curr. Appl. Phys. 2005, 5, $152-158$.

[9] C.P. Lee, J. D. Peng, D. Velayutham, J. Chang, P.W. Chen, V. Suryanarayanan, K.C. Ho, Electrochim. Acta 2013, 114, 303-308.

[10] M. Ue, M. Takeda, A. Toriumi, A. Kominato, R. Hagiwara, Y. Ito, J. Electrochem.Soc. 2003, 150, A499-A502.

[11] T. Sato, G. Masuda, K. Takagi, Electrochim. Acta 2004, 49, 3603-3611. 
[12] A. Stojanovic, C. Morgenbesser, D. Kogelnig, R. Krachler, B. K. Keppler Quaternary Ammonium and Phosphonium Ionic Liquids in Chemical and Environmental Engineering. In Ionic Liquids: Theory, Properties, New Approaches, (Ed.: A. Kokorin), InTech: Shangai, 2011, pp. $657-680$.

[13] B. Yu, D. G. Bansal, J. Qu, X. Sun, H. Luo, S. Dai, P. J. Blau, B. G. Bunting, G. Mordukhovich, D. J. Smolenski, Wear 2012, 289, 58-64.

[14] J. Weng, C. Wang, H. Li, J. Wang, Green Chem. 2006, 8, 96-99.

[15] K. J. Fraser, R. MacFarlane, Aust. J. Chem. 2009, 62, 309-321.

[16] C. J. Bradaric, A. Downard, C. Kennedy, A. J. Robertson, Y. Zhou, Green Chem. 2003, 5, 143152.

[17] V. Chakrapani, F. Rusli, M. A. Filler, P. A. Kohl, J. Phys. Chem. C 2011, 115, 22048-22053.

[18] N. W. Duffy, A. M. Bond, Electrochem. Commun. 2006, 8, 892-898.

[19] J. M. Pringle, D. R. MacFarlane, M. Forsyth, Synth. Met. 2005, 155, 684-689.

[20] E. Frackwiak, G. Lota, J. Pernak, Appl. Phys. Lett. 2005, 86, 164101-164103.

[21] R. E. Ramirez, E. M. Sanchez, Sol. Energy Mater. Sol. Cells 2006, 90, 2384-2390.

[22] R. E. Ramirez, L. C. Torres-Gonzalez, E. M. Sanchez, J. Electrochem. Soc. 2007, 154, B229-B233.

[23] K. Tsunashima, M. Sugiya, Electrochem Commun. 2007, 9, 2353-2358.

[24] C. L. S. Louros, A. F. M. Cláudio, C. M. S. S. Neves, M. G. Freire, I. M. Marrucho, J. Pauly, J. A. P. Coutinho, Int. J. Mol. Sci. 2010, 11, 1777-1791.

[25] R. J. Cornmell, C. L. Winder, S. Schuler, R. Goodacre, G. Stephens, Green Chem. 2008, 10, 685 691.

[26] C. M. S. S. Neves, J. F. O. Granjo, M. G. Freire, A. Robertson, N. M. C. Oliveira, J. A. P. Coutinho, Green Chem. 2011, 13, 1517-1526.

[27] P. J. Carvalho, I. Khan, A. Morais, J. F. O. Granjo, N. M. C. Oliveira, L. M. N. B. F. Santos, J. A. P. Coutinho, Fluid Phase Equilib. 2013, 354, 156-165.

[28] Y. Liu, L. Guo, L. Zhu, X. Sun, J. Chen, Chem. Eng. J. 2010, 158, 108-114.

[29] A. Stojanovic, D. Kogelnig, L. Fischer, S. Hann, M. Galanski, M. Groessl, R. Krachler, B. K. Keppler, Aust. J. Chem. 2010, 63, 511-524.

[30] P. J. Carvalho, V. H. Álvarez, I. M. Marrucho, M. Aznar, J. A. P. Coutinho, J. Supercrit. Fluids 2010, 52, 258-265.

[31] S. P. M. Ventura, J. Pauly, J. L. Daridon, J. A. Lopes da Silva, I. M. Marrucho, A. M. A. Dias, J. A. P. Coutinho, J. Chem. Thermodyn. 2008, 40, 1187-1192. 
[32] P. K. Kilaru, P. Scovazzo, Ind. Eng. Chem. Res. 2008, 47, 910-919.

[33] W. Zhang, S. Jiang, C. Hardacre, P. Goodrich, K. Wang, Z. Wu, H. Shao, Energy Fuels 2014, 28, 4333-4341.

[34] Y. Li, X. Wu, Q. Wu, D. Hong, W. Yan, Dalton Trans. 2014, 43, 13591-13595.

[35] H. J. Cristau, A. Long, H. Christol, Tetrahedron Lett. 1979, 20, 349-352.

[36] K. C. Lethesh, W. Dehaen, K. Binnemans, RSC Adv., 2014, 4, 4472-4477.

[37] D. Landini, A. Maia, A. Rampoldi, J. Org. Chem. 1986, 51, 3187-3191.

[38] M. Tariq, P. A. S. Forte, M. F. Costa Gomes, J. N. Canongia Lopes, L. P. N. Rebelo, J. Chem. Thermodyn. 2009, 41, 790-798.

[39] A. Stojanovic, D. Kogelnig, L. Fischer, S. Hann, M. Galanski, M. Grössl, R. Krachler, B. K. Keppler, Aust. J. Chem. 2010, 63, 511-524.

[40] J. Sun, D. R. MacFarlane, M. Forsyth, Ionics 1997, 3, 56-62.

[41] J. Sun, M. Forsyth, D. R. MacFarlane, J. Phys. Chem. B 1998, 102, 8858-8864.

[42] R. E. D. Sesto, C. Corley, A. Robertson, J. S. Wilkes, J. Organomet. Chem. 2005, 690, 25362542 .

[43] S. Seki, K. Hayamizu, S. Tsuzuki, K. Fujii, Y. Umebayashi, T. Mitsugi, T. Kobayashi, Y. Ohno, Y. Kobayashi, Y. Mita, H. Miyashiro, S.i. Ishiguro, Phys. Chem. Chem. Phys. 2009, 11, 3509-3514.

[44] H. Shirota, H. Fukazawa, T. Fujisawa, J. F. Wishart, J. Phys. Chem. B 2010, 114, 9400-9412.

[45] H. Y. Lee, H. Shirota, E. W. Castner, J. Phys. Chem. Lett. 2013, 4, 1477-1483.

[46] M. H. Ghatee, M. Bahrami, N. Khanjari, J Chem Thermodyn. 2013, 65, 42-52.

[47] R. K. Blundell, P. Licence, Phys.Chem.Chem.Phys. 2014, 16, 15278.

[48] P. J. Carvalho, S. P. M. Ventura, M. L. S. Batista, B. Schröder, F. Gonçalves, J. Esperança, F. Mutelet, J. A. P. Coutinho, J. Chem. Phys. 2014, 140, 064505.

[49] P. J. Griffin, A. P. Holt, K. Tsunashima, J. R. Sangoro, F. Kremer, A. P. Sokolov, J. Chem. Phys. 2015, 142, 084501-7.

[50] P. J. Griffin, A. P. Holt, Y. Wang, V. N. Novikov, J. R. Sangoro, F. Kremer, A. P. Sokolov, J. Phys. Chem. B 2014, 118, 783-790.

[51] T. Pott, P. Méléard, Phys. Chem. Chem. Phys. 2009, 11, 5469-5475.

[52] K. Tsunashima, Y. Sakai, M. Matsumiya, Electrochem. Commun. 2014, 39, 30-33.

[53] S. Seki, S. Tsuzuki, K. Hayamizu, N. Serizawa, S. Ono, K. Takei, H. Doi, Y. Umebayashi, J. Phys. Chem. B 2014, 118, 4590-4599.

[54] S. Pandian, S.G. Raju, K. S. Hariharan, S. M. Kolake, D. H. Park, M. J. Lee, J. Power Sources 2015, 286, 204-209. 
[55] L. K. Scarbath-Evers, P. A. Hunt, B. Kirchner, D. R. MacFarlane, S. Zahn, Phys. Chem. Chem. Phys. 2015, 17, 20205-20216.

[56] J. N. C. Lopes, A. A. H. Pádua, J. Phys. Chem. B 2004, 108, 16893-16898.

[57] L. J. A. Siqueira, M. C. C. Ribeiro, J. Phys. Chem. B 2007, 111, 11776-11785.

[58] L. J. A. Siqueira, M. C. C. Ribeiro, J. Phys. Chem. B 2009, 113, 1074-1079.

[59] S. Tsuzuki, W. Shinoda, H. Saito, M. Mikami, H. Tokuda, M. Watanabe, J. Phys. Chem. B 2009, 113, 10641-10649.

[60] O. Borodin, J. Phys. Chem. B 2009, 113, 11463-11478.

[61] K. Shimizu, A. A. H. Pádua, J. N. C. Lopes, J. Phys. Chem. B 2010, 114, 15635-15641.

[62] X. Liu, G. Z. S. Zhang, G. Yu, Molecular Simulation, 2010, 36, 79-86.

[63] C. S. Santos, H. V. R. Annapureddy, N. S. Murthy, H. K. Kashyap, E. W. J. Castner, C. J. Margulis, J. Chem. Phys. 2011, 134, 064501.

[64] H. K. Kashyap, C. S. Santos, H. V. R. Annapureddy, N. S. Murthy, C. J. Margulis, E. W. J. Castner, Faraday Discuss. 2012, 154, 133-143.

[65] H. K. Kashyap, C. S. Santos, R. P. Daly, J. J. Hettige, S. N. Murthy, H. Shirota, E. W. J. Castner, C. J. Margulis, J. Phys. Chem. B 2013, 117, 1130-1135.

[66] X. Liu, Y. Zhao, X. Zhang, G. Zhou, S. Zhang, J. Phys. Chem. B 2012, 116, 4934-4942.

[67] J. J. Hettige, H. K. Kashyap, C. J. Margulis, J. Chem. Phys. 2014, 140, 111102-1-4.

[68] M. Matsumiya, K. Hata, K. Tsunashima, J. Mol. Liq. 2015, 203,125-130.

[69] Y. U. Paulechka, G. J. Kabo, V. N. Emel'yanenko, J. Phys. Chem B 2008, 112, 15708-15717.

[70] Gaussian 03, Revision B.05, M.J. Frisch, G.W. Trucks, H.B. Schlegel, G. E.Scuseria, M.A. Robb, J.R. Cheeseman, J.A. Montgomery Jr., T. Vreven, K.N. Kudin, J.C. Burant, J.M. Millam, S.S. Iyengar, J. Tomasi, V. Barone, B. Mennucci, M. Cossi, G. Scalmani, N. Rega, G.A. Petersson, H. Nakatsuji, M. Hada, M. Ehara, K. Toyota, R. Fukuda, J. Hasegawa, M. Ishida, T. Nakajima, Y. Honda, O. Kitao, H. Nakai, M. Klene, X. Li, J.E. Knox, H.P. Hratchian, J.B. Cross, V. Bakken, C. Adamo, J. Jaramillo, R. Gomperts, R.E. Stratmann, O. Yazyev, A. J. Austin, R. Cammi, C. Pomelli, J.W. Ochterski, P.Y. Ayala, K. Morokuma, G.A. Voth, P. Salvador, J.J. Dannenberg, V. G. Zakrzewski, S. Dapprich, A.D. Daniels, M.C. Strain, O. Farkas, D.K. Malick, A.D. Rabuck, K. Raghavachari, J.B. Foresman, J.V. Ortiz, Q. Cui, A.G. Baboul, S. Clifford, J. Cioslowski, B.B. Stefanov, G. Liu, A. Liashenko, P. Piskorz, I. Komaromi, R.L. Martin, D.J. Fox, T. Keith, M.A. Al-Laham, C. Y. Peng, A. Nanayakkara, M. Challacombe, P.M.W. Gill, B. Johnson, W. Chen, M.W. Wong, C. Gonzalez, J.A. Pople, Gaussian Inc, Wallingford CT, 2004. 
[71] a) V. Barone, M. Cossi, J. Phys. Chem. A 1998, 102, 1995-2001; b) M. Cossi, N. Rega, G. Scalmani, V. Barone, J. Comput. Chem. 2003, 24, 669-681.

[72] G-E. Logotheti, J. Ramos, I. G. Economou, J. Phys. Chem. B, 2009, 113, 7211-7224.

[73] J. Schmidt, C. Krekeler, F. Dommert, Y. Zhao, R. Berger, L. D. Site, C. Holm, J. Phys. Chem. B 2010, 114, 6150-6155.

[74] M. H. Ghatee, A. R. Zolghadr, F. Moosavi, Y. Ansari, J. Chem. Phys. 2012, 136, 124706-14.

[75] J. N. A. C. Lopes, J. Deschamps, A. A. H. Padua, J. Phys. Chem. B 2004, 108, 2038-2047.

[76] J. N. A. C. Lopes, A. A. H. Padua, J. Phys. Chem. B 2006, 110, 19586-19592.

[77] J. N. A. C. Lopes, A. A. H. Padua, K. Shimizu, J. Phys. Chem. B 2008, 112, 5039-5046.

[78] M. P. Allen, D. J. Tildesley, Computer Simulation of Liquids; Clarendon Press: Oxford, UK, 1987.

[79] W. Smith, T. R. Forester, I. T. Todorov, The DL-POLY molecular simulation package, V. 2.17, Daresbury Laboratory, UK, 2006, www.cse.scitech.ac.uk/ccg/software/DL_POLY/; W. Smith, T. R. Forester, J. Mol. Graphics 1996, 14, 136.

[80] S. Tsuzuki, H. Matsumoto, W. Shinoda, M. Mikami, Phys. Chem. Chem. Phys. 2011, 13, 59875993.

[81] S. Tsuzuki, ChemPhysChem 2012, 13, 1664 - 1670.

[82] M. H. Ghatee, M. Zare, Ph.D. Thesis, Shiraz University, 2012.

[83] W. Jiang, T. Yan, Y. Wang, G. A. Voth, J. Phys. Chem. B 2008, 112, 3121-3131.

[84] Z. Hu, C. J. Margulis, J. Phys. Chem. B 2007, 111, 4705-4714.

[85] O. Borodin, G. D. Smith, J. Phys. Chem. B 2006, 110, 11481- 11490.

[86] M. H. Kowsari, S. Alavi, M. Ashrafizaadeh, B. Najafi, J. Chem. Phys. 2009, 130, 014703.

[87] S. Yeganegi, A. Soltanabadi, D. Farmanzadeh, J. Phys. Chem. B 2012, 116, 11517-11526.

[88] F. Dommert, K. Wendler, R. Berger, L. D. Site, C. Holm, Chemphyschem 2012, 13, 1625-1637.

[89] W. Zhao, F. Leroy, B. Heggen, S. Zahn, B. Kirchner, S. Balasubramanian, F. Muller-Plathe, J. Am. Chem. Soc. 2009, 131, 15825-15833.

[90] M. Kohagen, M. Brehm, J. Thar, W. Zhao, F. Muller-Plathe, B. Kirchner, J. Phys. Chem. B 2011, $115,693-702$.

[91] Y. Zhang, E. J. Maginn, J. Phys. Chem. Lett. 2015, 6, 700-705.

[92] J. N. A. C. Lopes, A. A. H. Pádua, J. Phys. Chem. B 2006, 110, 3330-3335.

[93] K. Shimizu, M. F. C. Gomes, A. A. H. Pádua, L. P. N. Rebelo, J. N. C. Lopes, J. Mol. Struct. 2010, 946, 70-76.

[94] M. Brehm, B. Kirchner, J. Chem. Inf. Model 2011, 51, 2007-2023. 